IMA Journal of Applied Mathematics (2020) Page 1 of 24

doi:10.1093/imamat/xxx000

\title{
A stacked frequency approach for inhomogeneous time-dependent MRE: an inverse problem for the elastic shear modulus
}

\section{FINAL VERSION: October 15, 2020}

\author{
PENNY J DAVIES* \\ Department of Mathematics and Statistics, University of Strathclyde, 26 Richmond St, \\ Glasgow, G1 1XH, UK \\ *Corresponding author: penny.davies@strath.ac.uk \\ AND \\ INGOLF SACK \\ Department of Radiology, Charité-Universitätsmedizin, Berlin, Germany \\ ingolf.sack@charite.de
}

[Received on 15 October 2020]

\begin{abstract}
We derive and analyse a new way to calculate the shear modulus of an inhomogeneous elastic material from time-dependent MRE (magnetic resonance elastography) measurements of its interior displacement. Even with such a rich data source this is a challenging inverse problem because the coefficient of the shear modulus in the governing equations can be small (or potentially zero). Our approach overcomes this by combining different data sets into an overdetermined matrix-vector equation. It uses finite differences to approximate space derivatives and a Fourier interpolant in time, and we do not need to assume that the inhomogeneous material is 'locally homogeneous'. Crucially, our construction ensures that the computed value of the (real) shear modulus is real: approximation methods based on the frequency domain version of the problem often give a complex shear modulus for the elastic case and this can be hard to interpret, especially if its imaginary part dominates. We carry out careful numerical tests on a one (space) dimensional analogue of the problem, and on experimental MRE data for an inhomogeneous gel phantom.
\end{abstract}

Keywords:

Magnetic resonance elastography (MRE), elasticity, biomechanics, inverse problem, time-dependent

2000 Math Subject Classification: 74L15, 74530, 35R30

\section{Introduction}

Magnetic resonance elastography (MRE) is a powerful technique for non-invasive determination of the biomechanical properties (such as the elastic shear modulus) of tissue. A common experimental scenario is to use an external mechanical excitation to induce waves in the tissue, and measure the resulting displacement at fixed sites inside the tissue using phase-contrast MRI. This provides a far richer data source than surface-based mechanical tests, but accurate reconstruction of the elastic moduli is still a challenging ill-conditioned inverse problem, with many different methods proposed (see e.g. Manduca et al. (2001); Park \& Maniatty (2006); McLaughlin et al. (2010); Sánchez et al. (2010); Doyley (2012); Dittmann et al. (2016); Barnhill et al. (2018)).

Two aspects of this problem were recently considered in Davies et al. (2019). One is a derivation and 
analysis of a new method (termed "stacked frequency wave inversion", or SFWI) to calculate the shear modulus of an inhomogeneous elastic material from time-harmonic measurements of the material's displacement (i.e. the frequency domain version of the MRE problem). The underlying problem is ill-conditioned because the coefficient of the shear modulus in the governing equations can be zero or small, and the SFWI approach overcomes this by combining approximations at different mechanical oscillation frequencies into a single overdetermined matrix-vector equation. The other aspect addressed in Davies et al. (2019) is how to use the full time signal of measured displacements rather than a single Fourier component (as is standard) to calculate the shear modulus of a homogeneous material.

Here we derive a new stacked frequency approximation method for the full time-dependent and inhomogeneous problem which automatically gives a real value for the (real, nonconstant) shear modulus in the pure elastic case. This is important because any method which gives a complex value for the (pure elastic) shear modulus is unlikely to be robust enough to use for more complicated viscous materials. Our approach uses finite differences to approximate space derivatives and a Fourier interpolant in time and does not rely on assuming that the inhomogeneous material is 'locally homogeneous'.

We consider two variants of the new method, one which uses all the information present in the timesignal, and another based on the dominant Fourier component from the measured displacement - this can be advantageous in experiments with low frequency machine noise. These new methods are tested extensively on "synthetic" data with added noise and on experimental data. In both cases we assume that the tissue's displacement is measured at uniformly spaced points (or as averages over equal-sized pixels or voxels) in $\Omega_{L} \subset \mathbb{R}^{d}$ for $d=2$ or 3 , where $L$ is a typical length scale - in applications $\Omega_{L}$ is a rectangle or box with sides of length $\mathscr{O}(L)$, and at $M$ times during a complete period of the underlying mechanical oscillation (in practice $M=8$ ).

The displacement $\mathbf{U}(\mathbf{X}, \tau)$ of an elastic material at time $\tau$ of a point originally at $\mathbf{X} \in \Omega_{L}$ satisfies the linear momentum balance equation $\rho \partial_{\tau}^{2} \mathbf{U}=\operatorname{div} T$ (assuming that body forces such as gravity are small enough to be ignored), where $\rho$ is the material's density, $\partial_{\tau}$ denotes the total derivative with respect to $\tau$ and $T$ is the stress tensor corresponding to the displacement $\mathbf{U}$. We assume that: the displacement $\mathbf{U}$ is small enough for a linear model to be valid; the material is isotropic (i.e. there is not a preferred direction, unlike muscle fibres); and phenomena such as wave attenuation are ignored (see the end of Sec 2.3 for a discussion of the behaviour of viscous models). In this case the stress is $T_{\alpha \beta}=\lambda U_{\gamma, \gamma} \delta_{\alpha \beta}+\tilde{\mu}\left(U_{\alpha, \beta}+U_{\beta, \alpha}\right)$, where ${ }_{, \alpha}=\partial / \partial X_{\alpha}$, and $\lambda(\mathbf{X})$ and $\tilde{\mu}(\mathbf{X})$ are Lamé material parameters ( $\tilde{\mu}$ is the shear modulus), and the displacement satisfies the elastic wave equation (EWE)

$$
\rho \partial_{\tau}^{2} U_{\alpha}=\left(\tilde{\mu}\left(U_{\alpha, \beta}+U_{\beta, \alpha}\right)\right)_{\beta}+\left(\lambda U_{\beta, \beta}\right), \alpha \quad \text { for } \alpha=1: d .
$$

The EWE admits longitudinal (pressure) and transverse (shear) plane wave solutions, but at the typical frequencies used in MRE (up to a few hundred $\mathrm{Hz}$ ), the pressure wave has a wavelength of the order of several metres, which is undetectable. It is typical to filter the displacement to remove the gradient part of $\mathbf{U}$ in MRE applications (see e.g. Manduca et al. (2001)), resulting in the shear wave equation (SWE)

$$
\rho \partial_{\tau}^{2} U_{\alpha}=\left(\tilde{\mu}\left(U_{\alpha, \beta}+U_{\beta, \alpha}\right)\right), \beta \quad \text { for } \alpha=1: d,
$$

where now $\operatorname{div} \mathbf{U}=0$.

Our MRE reconstruction problem is then to determine the (inhomogeneous) shear modulus $\tilde{\mu}$ from (1.2) using space-time measurements of $\mathbf{U}$. We first nondimensionalize the SWE (1.2), setting:

$$
\mathbf{x}=\mathbf{X} / L, t=2 \pi f \tau, \mu(\mathbf{x})=\tilde{\mu}(\mathbf{X}) / \mu_{0}, \text { and } \mathbf{u}(\mathbf{x}, t)=\mathbf{U}(\mathbf{X}, \tau),
$$


where $\mu_{0}$ is a constant of the same order of magnitude as $\tilde{\mu}$, so that $\mu(\mathbf{x})$ is an $\mathscr{O}(1)$ quantity. This gives the nondimensionalized SWE

$$
\omega^{2} \ddot{u}_{\alpha}=\left(\mu\left(u_{\alpha, \beta}+u_{\beta, \alpha}\right)\right), \beta \quad \text { for } \alpha=1: d, \text { for }(\mathbf{x}, t) \in \Omega_{1} \times(0,2 \pi),
$$

where,$\alpha$ now denotes the partial derivative with respect to $x_{\alpha}$, an overdot denotes a derivative with respect to $t$ and

$$
\omega=2 \pi f L \sqrt{\frac{\rho}{\mu_{0}}} .
$$

The MRE problem in 1D space (i.e. the 1D analogue of (1.3)) provides a very useful test case because it behaves in a qualitatively similar way to MRE in higher spatial dimension, but is far easier and quicker to simulate. We first derive and analyze two variants of the time domain SFWI algorithm for the 1D MRE problem and carefully evaluate their performance in the presence of added noise. To do this we need to produce highly accurate displacement data for the $1 \mathrm{D}$ version of the time-dependent equation (1.3), and this is described at the start of Section 2.

Results for the two SFWI algorithms on experimental MRE measurements for a gel phantom are given in Section 3. As in 1D the solution accuracy is significantly degraded by measurement noise, and we consider noise further in Section 4, and conclude in Section 5 with some pragmatic suggestions for MRE practitioners.

\section{Approximation in 1D space}

As noted in Davies et al. (2019), 1D simulations are very useful in providing qualitatively similar results to those for higher space dimensions, whilst having the advantage of being much faster calculations. It is also far more straightforward to produce exact or highly accurate "synthetic" test data in 1D, which is needed in order to evaluate how well a reconstruction algorithm behaves with increasing noise. The 1D model problem is to find $\mu(x)$ given the nondimensionalized frequency $\omega$ and measured values of the time periodic function $u(x, t)$ such that

$$
\omega^{2} \ddot{u}=\left(\mu u_{x}\right)_{x}, \quad x \in(0,1), \quad t \in(0,2 \pi),
$$

where the subscript ${ }_{x}$ denotes $\partial / \partial x$. We describe "stacked frequency" inversion algorithms for this timedependent inhomogeneous problem in Sec. 2.3, but first show how test data can be obtained efficiently.

\subsection{Construction of synthetic data for the $1 D$ problem (2.1)}

It is straightforward to calculate an exact solution $u(x, t)$ of $(2.1)$ when $\mu(x)$ is piecewise constant with one jump. Suppose that

$$
\mu(x)= \begin{cases}1, & x \in[0, a] \\ \mu_{I}>0, & x \in(a, 1]\end{cases}
$$

for some $a \in(0,1)$ and let the wave $\phi(t-\omega(x-a))$ be incident upon the material for $x<a$. It travels through the material undisturbed until it hits the material discontinuity at $x=a$, which generates a reflected wave with the same speed (and opposite direction) in $x<a$ and a transmitted wave with a different speed in $x>a$. Maintaining continuity of $u$ and the stress $\mu u_{x}$ at $x=a$ gives the solution as

$$
u(x, t)= \begin{cases}\phi(t-\omega(x-a))+K_{R} \phi(t+\omega(x-a)), & x \in[0, a] \\ K_{T} \phi\left(t-\omega_{I}(x-a)\right), & x \in(a, 1],\end{cases}
$$


where $\omega_{I}=\omega / \sqrt{\mu_{I}}$ and the reflection and transmission coefficients are respectively

$$
K_{R}=\frac{1-\sqrt{\mu_{I}}}{1+\sqrt{\mu_{I}}} \quad \text { and } \quad K_{T}=1+K_{R}=\frac{2}{1+\sqrt{\mu_{I}}} .
$$

The incident wave is assumed to be $2 \pi$-periodic in time, and for simplicity we take $\phi(t)=\cos t$. This gives the solution $u(x, t)=v_{c}(x) \cos t+v_{s}(x) \sin t$, where

$v_{c}(x)=\left\{\begin{array}{ll}\left(1+K_{R}\right) \cos (\omega(x-a)), & x \in[0, a] \\ K_{T} \cos \left(\omega_{I}(x-a)\right), & x \in(a, 1],\end{array} \quad\right.$ and $\quad v_{s}(x)= \begin{cases}\left(1-K_{R}\right) \sin (\omega(x-a)), & x \in[0, a] \\ K_{T} \sin \left(\omega_{I}(x-a)\right), & x \in(a, 1] .\end{cases}$

Note that both $v_{c}(x)$ and $v_{s}(x)$ solve the ODE

$$
\left(\mu v^{\prime}\right)^{\prime}+\omega^{2} v=0
$$

where' denotes $d / d x$, and this can be regarded as an initial value problem (IVP) when the pairs of initial conditions are

$$
\begin{aligned}
& v_{c}(0)=\left(1+K_{R}\right) \cos (\omega a) \\
& v_{c}^{\prime}(0)=\omega\left(1+K_{R}\right) \sin (\omega a)
\end{aligned} \text { and } \begin{aligned}
v_{s}(0) & =\left(K_{R}-1\right) \sin (\omega a) \\
v_{s}^{\prime}(0) & =\omega\left(1-K_{R}\right) \cos (\omega a) .
\end{aligned}
$$

This formulation is the most useful way to construct highly accurate numerical solutions $u(x, t)$ of $(2.1)$ when the exact shear modulus $\mu(x)$ is more complicated than a piecewise constant with a single jump, because of the wide availability of efficient ODE solvers for IVPs. In standard first order form the ODE (2.2) for $\mathbf{y}=\left(y_{1}, y_{2}\right)^{T}$ with $y_{1}=v$ and $y_{2}=\mu v^{\prime}$ is

$$
\mathbf{y}^{\prime}(x)=\left(\begin{array}{cc}
0 & 1 / \mu(x) \\
-\omega^{2} & 0
\end{array}\right) \mathbf{y}(x)
$$

and this is straightforward to solve numerically with high accuracy to obtain solutions $\mathbf{y}^{(I)}(x)$ and $\mathbf{y}^{(I I)}(x)$ with the initial conditions $\mathbf{y}^{(I)}(0)=(1,0)^{T}$ and $\mathbf{y}^{(I I)}(0)=(0,1)^{T}$ respectively. Any solution of the ODE (2.2) with specified initial conditions can then be written as a linear combination of the first components of these solutions. For example,

$$
v(x)=v_{0} y_{1}^{(I)}(x)+v_{1} \mu(0) y_{1}^{(I I)}(x)
$$

solves (2.2) with initial conditions $v(0)=v_{0}$ and $v^{\prime}(0)=v_{1}$. Numerical test results indicate that this approach works well, giving very accurate test data $v(x)$.

Our strategy for obtaining synthetic test data to use for the time-dependent shear modulus problem in $1 \mathrm{D}$ space is as follows.

- Choose the exact $\mu(x)>0$ for $x \in[0,1]$ which the MRE simulation is to approximate and a suitable non-dimensionalised frequency $\omega$.

- Use a standard highly accurate ODE solver to evaluate the solutions $\mathbf{y}^{(I)}$ and $\mathbf{y}^{(I I)}$ of (2.3) on an extremely fine spatial mesh. The function $u(x, t)=v(x) \cos t$ with $v(x)$ given by (2.4) on this mesh for given values of $v_{0}$ and $v_{1}$ approximates the solution of the PDE (2.1) with initial and boundary conditions

$$
u(x, 0)=v(x), \quad u_{t}(x, 0)=0, \quad u(0, t)=v_{0} \cos t, \quad u_{x}(0, t)=v_{1} \cos t .
$$


- Choose space-time "observation mesh" sizes $\Delta x=1 / J$ and $h=2 \pi / M$ and produce a noisy version of $u$ on this mesh, setting

$$
u_{j}^{m}=v\left(x_{j-1 / 2}\right) \cos \left(t_{m}\right)+\varepsilon_{j}^{m}, \quad j=1: J, m=0: M-1,
$$

where $x_{r}=r \Delta x, t_{m}=m h$ and each $\varepsilon_{j}^{m} \in[-\varepsilon, \varepsilon]$ for $\varepsilon \geqslant 0$ is a pseudo-random error term to simulate experimental error.

The aim of the reconstruction algorithm is to combine synthetic data values obtained at more than one "frequency" $\omega$ in order to approximate the shear modulus. Our new time domain algorithm is based on the spatial SFWI derived in Davies et al. (2019) for time harmonic (frequency domain) data and uses the Fourier interpolant of $u$ in time. This construction, which follows the approach of Trefethen (2000), is used for the homogeneous (i.e. constant $\mu$ ) problem in Davies et al. (2019) and is summarised below.

\subsection{Fourier interpolant in time}

Suppose that $v \in C[0,2 \pi]$ is a $2 \pi$-periodic function measured at $t_{m}$ for $m=0: M-1$, and set $v^{m}=v\left(t_{m}\right)$. The discrete Fourier transform (DFT) of $v$ is $\left\{\widehat{v}^{\ell}: \ell=-M / 2+1: M / 2\right\}$, where

$$
\widehat{v}^{\ell}=\sum_{m=0}^{M-1} e^{-i \ell t_{m}} v^{m}, \quad \ell=-M / 2+1: M / 2
$$

and the inverse DFT is

$$
v^{m}=\frac{1}{M} \sum_{\ell=-M / 2}^{M / 2} e^{i \ell t_{m}} \widehat{v}^{\ell}, \quad m=0: M-1
$$

where $\widehat{v}^{-M / 2}=\widehat{v}^{M / 2}$ and the prime on the sum indicates that terms with $\ell= \pm M / 2$ are multiplied by $\frac{1}{2}$. The Fourier interpolant of $v$ is $V(t)$ defined by

$$
V(t)=\frac{1}{M} \sum_{\ell=-M / 2}^{M / 2} e^{i \ell t} \widehat{v}^{\ell}
$$

and satisfies $V\left(t_{m}\right)=v\left(t_{m}\right)$ for each $m$ by construction. Differentiating twice in time at $t=t_{m}$ gives

$$
\ddot{V}\left(t_{m}\right)=\frac{1}{M} \sum_{\ell=-M / 2+1}^{M / 2}\left(-\ell^{2}\right) e^{i \ell t_{m}} \widehat{v}^{\ell}, \quad m=0: M-1 .
$$

As noted in Ch. 3 of Trefethen (2000), if the DFT is implemented in a way which assumes a different ordering of the wavenumbers then the vector of multipliers of the components $-\ell^{2}$ will also need to be reordered. For example, if using MATLAB's $\mathrm{ft}$ function then the vector of multipliers of the components $e^{i \ell t_{m}}$ in (2.6) is $[0: \mathrm{M} / 2,-\mathrm{M} / 2+1: 1] \cdot{ }^{\wedge} 2 . * \mathrm{fft}(\mathrm{v})$.

Note that if $v$ is a pure harmonic function, i.e. $v(t)=A \cos (t+c)$ for $A, c \in \mathbb{R}$, then $\widehat{v}^{\ell}=0$ unless $\ell= \pm 1$. For an MRE signal (in which there is experimental noise) the Fourier components with $\ell= \pm 1$ correspond to the underlying oscillation frequency and will be larger than the others, and we refer to them as the dominant Fourier components. They are complex conjugates - the positive and negative fundamental frequencies in the Fourier spectrum. 


\subsection{Time-dependent stacked frequency wave inversion method for (2.1)}

We now describe and analyze a new approximation algorithm for the inhomogeneous and time-dependent MRE reconstruction problem (2.1), which is the 1D analogue of a first order hyperbolic PDE for $\mu$. The displacement $u$ has an underlying time-harmonic oscillation and it is quite likely that the 'wind' $\left(u_{x}\right.$ in (2.1)) will change sign, and its sign cannot be determined accurately because $u_{x}$ is not measured directly, but has to be approximated from measured values of $u$. This makes an upwinding approximation scheme for (2.1) and its higher dimensional versions inappropriate, and instead we use central differences for the space approximation, as described in Davies et al. (2019), regarding $u_{j+1 / 2}^{m}$ as the average value of $u$ over the space-interval $\left[x_{j}, x_{j+1}\right]$ at time $t^{m}$. The gridpoints for $\mu$ are $x_{j}$ for $j=1: J-1$, i.e. the endpoints of interior intervals $\left(x_{j}, x_{j+1}\right)$.

Integrating (2.1) over such an interval for $j=1: J-2$ gives

$$
\left[\mu(x) u_{x}(x, t)\right]_{x_{j}}^{x_{j+1}}=\omega^{2} \int_{x_{j}}^{x_{j+1}} \ddot{u}(x, t) d x \approx \omega^{2} \Delta x \ddot{u}\left(x_{j+1 / 2}, t\right)
$$

using the midpoint rule. We use central differences for $u_{x}$ terms: $u_{x}\left(x_{j}, t\right) \approx\left(u\left(x_{j+1 / 2}, t\right)-u\left(x_{j-1 / 2}, t\right)\right) / \Delta x$ and replace $u$ by its Fourier interpolant in time. This gives the following approximation scheme

$$
a_{j+1}^{\ell} \mu_{j+1}-a_{j}^{\ell} \mu_{j} \approx \omega^{2} \Delta x\left(-\ell^{2}\right) \widehat{u}_{j+1 / 2}^{\ell}, \quad j=1: J-2, \quad \ell=-M / 2+1: M / 2,
$$

where

$$
\widehat{u}_{j+1 / 2}^{\ell}=\sum_{m=0}^{M-1} e^{-i \ell t_{m}} u\left(x_{j+1 / 2}, t_{m}\right) \quad \text { and } \quad a_{j}^{\ell}=\frac{\left(\widehat{u}_{j+1 / 2}^{\ell}-\widehat{u}_{j-1 / 2}^{\ell}\right)}{\Delta x} .
$$

Note that the right-hand side of (2.8) is zero when $\ell=0$ (this zero frequency corresponds to $u$ being constant) and we ignore this mode when writing the equations as a linear system, setting $\mathbf{a}_{j}=$ $\left(a_{j}^{-M / 2+1}, \ldots, a_{j}^{-1}, a_{j}^{1}, \ldots, a_{j}^{M / 2}\right)^{T} \in \mathbb{C}^{M-1}, \mathbf{0} \in \mathbb{R}^{M-1}$ to be the zero (column) vector and defining $A \in$ $\mathbb{C}^{(M-1)(J-2) \times(J-1)}$ to be the block bidiagonal matrix with entries

$$
A=\left(\begin{array}{ccccccc}
\mathbf{a}_{1} & -\mathbf{a}_{2} & \mathbf{0} & \mathbf{0} & \ldots & \mathbf{0} & \mathbf{0} \\
\mathbf{0} & \mathbf{a}_{2} & -\mathbf{a}_{3} & \mathbf{0} & \ldots & \mathbf{0} & \mathbf{0} \\
\vdots & \vdots & \ddots & \ddots & \ddots & \ddots & \vdots \\
\mathbf{0} & \mathbf{0} & \mathbf{0} & \mathbf{0} & \ldots & \mathbf{a}_{J-2} & -\mathbf{a}_{J-1}
\end{array}\right)
$$

Then the approximation (2.8) is the underdetermined linear system

$$
A \mu=\mathbf{b}
$$

for $\mu=\left(\mu_{1}, \ldots, \mu_{J-1}\right)^{T}$, where $\mathbf{b} \in \mathbb{C}^{(M-1)(J-2)}$ has components $-\omega^{2} \ell^{2} \Delta x \widehat{u}_{j-1 / 2}^{\ell}$ for $\ell=-M / 2+1$ : $M / 2, \ell \neq 0$ and $j=1: J-2$, stacked vertically in the appropriate order. Note the difference in structure from the equivalent linear system for the time-harmonic version of (2.1) in Davies et al. (2019): the matrix $A$ here has complex (rather than real) entries, and has $M-1$ times the number of rows. But the fundamental issues remain the same: (i) the linear system (2.8) is underdetermined because as a first order ODE for $\mu(x),(2.1)$ is only enough to give the general solution to within an arbitrary constant, and needs an extra piece of information (such as a boundary condition) to give a unique solution, and (ii) it is likely to be ill conditioned because the $a_{j}^{\ell}$ can be close to zero. For this reason we combine $q>1$ 
systems like (2.9), each obtained from values of $u(x, t)$ at different nondimensional frequencies $\omega_{k}$ to produce a least squares (overdetermined) system for $\mu$. Suppose that they individually give the systems $A^{(k)} \mu=\mathbf{b}^{(k)}$ for $k=1: q$. Setting

$$
\tilde{A}=\left[\begin{array}{c}
A^{(1)} \\
\vdots \\
A^{(q)}
\end{array}\right] \quad \text { and } \quad \tilde{\mathbf{b}}=\left[\begin{array}{c}
\mathbf{b}^{(1)} \\
\vdots \\
\mathbf{b}^{(q)}
\end{array}\right]
$$

gives the least squares formulation

$$
\min _{\mu \in \mathbb{C}^{J-1}}\|\tilde{A} \mu-\tilde{\mathbf{b}}\|
$$

where $\|\cdot\|$ is the (complex) 2-norm, $\|\mathbf{c}\|=\sqrt{\mathbf{c}^{H} \mathbf{c}}$. We first show that the structure of the individual matrices $A^{(k)}$ guarantees that the least squares solution $\mu_{\mathrm{LS}}$ of (2.11) is real. It satisfies

$$
\tilde{A}^{H} \tilde{A} \mu_{\mathrm{LS}}=\tilde{A}^{H} \tilde{\mathbf{b}}
$$

where the block structure of $\tilde{A}$ and $\tilde{\mathbf{b}}$ gives

$$
\tilde{A}^{H} \tilde{A}=\sum_{k=1}^{q}\left(A^{(k)}\right)^{H} A^{(k)} \quad \text { and } \quad \tilde{A}^{H} \tilde{\mathbf{b}}=\sum_{k=1}^{q}\left(A^{(k)}\right)^{H} \mathbf{b}^{(k)} .
$$

We look at a single term from each sum (dropping the superscript ${ }^{(k)}$ for convenience). The $j$ th diagonal entry of the $(J-1) \times(J-1)$ tridiagonal matrix $A^{H} A$ is $\alpha_{j}\left|\mathbf{a}_{j}\right|^{2}$ where $\alpha_{1}=\alpha_{J-1}=1$ and all other $\alpha_{j}=2$, and hence all diagonal entries are real and non-negative. Each nonzero off-diagonal is equal to $-\mathbf{a}_{j}^{H} \mathbf{a}_{j+1}$ or its complex conjugate, and

$$
-\mathbf{a}_{j}^{H} \mathbf{a}_{j+1}=\frac{-1}{\Delta x^{2}} \sum_{|\ell|=1}^{M / 2}\left(\overline{\widehat{u}_{j+1 / 2}^{\ell}-\widehat{u}_{j-1 / 2}^{\ell}}\right)\left(\widehat{u}_{j+3 / 2}^{\ell}-\widehat{u}_{j+1 / 2}^{\ell}\right) .
$$

Because $\overline{\widehat{u}}_{p}^{\ell}=\widehat{u}_{p}^{\ell}$ it follows that each term in the above sum can be written as

$$
\sum_{|\ell|=1}^{M / 2} \overline{\widehat{u}}_{p}^{\ell} \widehat{u}_{r}^{\ell}=2 \sum_{\ell=1}^{M / 2} \Re\left(\overline{\widehat{u}}_{p}^{\ell} \widehat{u}_{r}^{\ell}\right) \in \mathbb{R}
$$

Thus all components of $A^{H} A$ (and hence also of $\tilde{A}^{H} \tilde{A}$ ) are real. It can similarly be shown that the components of $A^{H} \mathbf{b}$ involve sums of the following form:

$$
\sum_{|\ell|=1}^{M / 2} \ell^{2} \widehat{\widehat{u}}_{p}^{\ell} \widehat{u}_{r}^{\ell}=2 \sum_{\ell=1}^{M / 2} \ell^{2} \Re\left(\overline{\widehat{u}}_{p}^{\ell} \widehat{u}_{r}^{\ell}\right) \in \mathbb{R}
$$

and so are also real. This means that both the matrix and right-hand side vector in (2.12) are real, which guarantees that $\mu_{\mathrm{LS}}$ is real in exact arithmetic (and it is real up to rounding error in numerical experiments).

A common approach in experimental applications is to just use the dominant Fourier component with $\ell=1$ (corresponding to the underlying oscillation frequency) in building each $A^{(k)}$ and $\mathbf{b}^{(k)}$ of 
(2.10). In this case the least squares solution $\mu_{\mathrm{LS}}$ is complex, and it can be hard to understand what it actually represents (especially as the imaginary part can be dominant, as shown in Fig. 3.4 of Davies et al. (2019) for a homogeneous material). However there can be very good reasons for only using the dominant frequency, such as wanting to prevent lower frequency machine noise from contaminating the calculation. We note that if the two dominant components from the DFT are used (they are complex conjugates corresponding to $\ell= \pm 1$ in (2.8)), then the least squares solution in this case is again real (up to rounding error) and we denote it by $\mu_{\mathrm{LS}}^{*}$.

Papers in the MRE literature frequently refer to the shear modulus $\mu$ as being complex, e.g. Sec. 5.2 of Manduca et al. (2001) states that its imaginary part represents "attenuation for a viscoelastic medium". This description can be misleading, especially for a multi-frequency approximation. As described in ?, linear viscoelastic materials can be modelled by equations of motion which include extra time derivative terms, e.g.

$$
\omega^{2} \ddot{u}=\left(\mu u_{x}+v \dot{u}_{x}\right)_{x}, \quad x \in(0,1), \quad t \in(0,2 \pi),
$$

might be used instead of (2.1) in $1 \mathrm{D}$, where both $\mu(x)$ and $v(x)$ are real quantities. Taking the DFT gives the equation

$$
-\omega^{2} \ell^{2} \widehat{u}^{\ell}=\left((\mu+i \omega \ell v) \widehat{u}_{x}^{\ell}\right)_{x}
$$

for the $\ell$ th Fourier component, and so the complex coefficient $\mu+i \omega \ell v$ is playing the same role for the damped model (2.13) as $\mu$ does for (2.1). But this is not the same as starting with the elastic equation (2.1) and then interpreting the imaginary part of the calculated shear modulus as being due to damping or attenuation, when it could just be due to an inaccurate algorithm, as illustrated for the single mode case in Fig. 3.4 of Davies et al. (2019).

We do not consider viscoelastic models further here, and now evaluate the accuracy of our two variants of the SFWI algorithm in approximating a known $\mu(x)$ for the elastic case (2.1).

\subsection{Numerical results for synthetic $1 D$ data}

We now give numerical test results for the two variants of the SFWI approximation of the inhomogeneous 1D time-dependent problem (2.1) described above. These 1D results allow us to in quantify the algorithms' behaviour in the presence of noise in a way which cannot be easily done in higher space dimensions (where it is hard to obtain highly accurate test solutions). The two algorithms are:

SFWI-1: uses $M-1$ Fourier components for the matrices $A^{(k)}$ and vectors $\mathbf{b}^{(k)}$ in (2.10), with the solution of (2.11) denoted by $\mu_{\mathrm{LS}}$;

SFWI-2: uses the two dominant Fourier components $(\ell= \pm 1)$ for the matrices $A^{(k)}$ and vectors $\mathbf{b}^{(k)}$ in (2.10), with the solution of (2.11) denoted by $\mu_{\mathrm{LS}}^{*}$.

We begin with the same example used in Davies et al. (2019) for the frequency domain version of the problem, to aid comparison with that case, and assume that the (exact) $\mu(x)$ is piecewise constant on $[0,1]$ with

$$
\mu(x)= \begin{cases}1, & x \in[0, a) \cup(b, 1] \\ \mu_{I}, & x \in(a, b)\end{cases}
$$

with $a=0.35, b=0.69$ and $\mu_{I}=3$, and use the non-dimensionalised frequencies $\omega_{k}=[40: 10: 80]$. Results for $\mu_{\mathrm{LS}}$ with $J=64$ space meshpoints are shown in Figure 2.1, with each plot giving the results of 100 simulations at added noise level $\varepsilon=0.02$ (top) and $\varepsilon=0.1$ (bottom), and results for $\mu_{\mathrm{LS}}^{*}$ are 
qualitatively very similar. Both algorithms perform well on this mesh, even with $10 \%$ added noise. In all cases the imaginary component of the calculated shear modulus is at the level of rounding error, and is ignored.
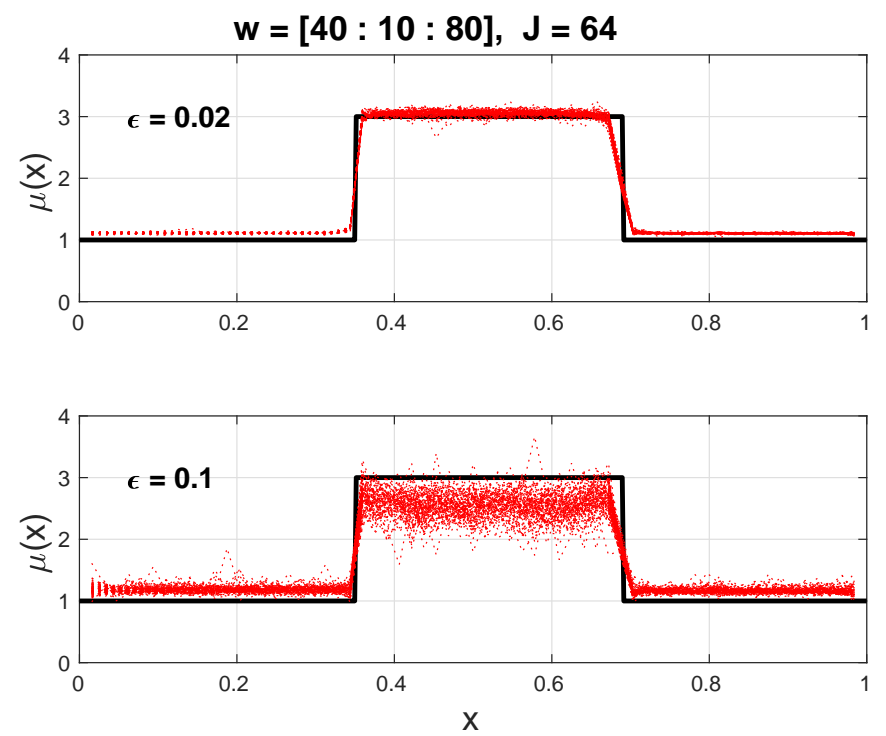

FIG. 2.1. Plot of 100 simulations of calculated $\mu_{\mathrm{LS}}$ from SFWI-1 (red dotted lines) obtained using $\omega_{k}=[40: 10: 80]$ when $J=64$ and at noise-levels $\varepsilon=0.02$ (top) and $\varepsilon=0.1$ (bottom). The exact value $\mu_{\mathrm{ex}}$ is shown as a solid black line.

Both algorithms' performance degrades surprisingly quickly as the mesh is refined, and Figures 2.2-2.3 are plots for $\mu_{\mathrm{LS}}$ and $\mu_{\mathrm{LS}}^{*}$ respectively, when $J=128$. The error in the calculated solution is noticeable even at $2 \%$ error (although SFWI-2 works better than SFWI-1), and there is essentially no evidence of the inclusion when $\varepsilon=0.1$.

The $L^{1}$-norms of the error in $\mu_{\mathrm{LS}}$ and $\mu_{\mathrm{LS}}^{*}$ are respectively defined as

$$
\mathscr{E}_{1}=\frac{1}{J-1} \sum_{j=1}^{J-1}\left|\left(\mu_{\mathrm{LS}}-\mu_{\mathrm{ex}}\right)_{j}\right| \quad \text { and } \quad \mathscr{E}_{1}^{*}=\frac{1}{J-1} \sum_{j=1}^{J-1}\left|\left(\mu_{\mathrm{LS}}^{*}-\mu_{\mathrm{ex}}\right)_{j}\right|
$$

where $\mu_{\mathrm{ex}}$ is the vector $\left\{\mu\left(x_{j}\right)\right\}_{j=1}^{J-1}$. Figure 2.4 shows summary plots of these $L^{1}$ errors at a range of values of $\varepsilon$. In all cases the rapid growth in error (as the noise level $\varepsilon$ increases) corresponds to the computed solution being less than the exact solution, as illustrated in e.g. Fig. 2.2. The components of $\tilde{A}$ are differences whose error is proportional to $\varepsilon / \Delta x$, and so it is not surprising that the error increases as the mesh is refined. It is well known that noisy MRE data can cause the computed shear modulus to be severely underestimated (see e.g. Arunachalam et al. (2017)) but it is not obvious why the error grows so rapidly, and we consider this further in Sec. 4. 

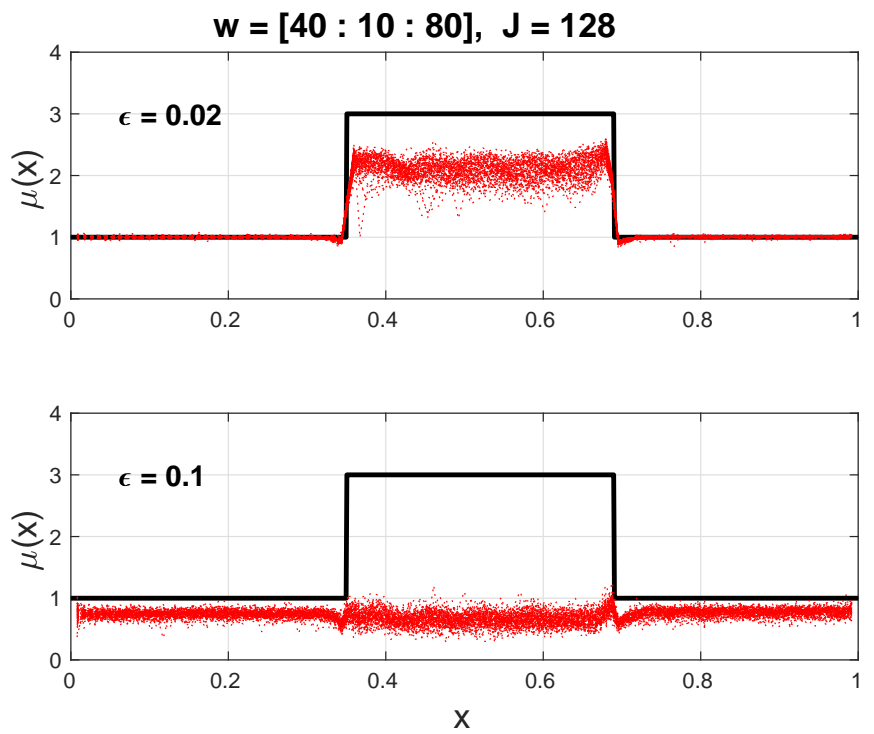

FIG. 2.2. Plot of 100 simulations of calculated $\mu_{\mathrm{LS}}$ from SFWI-1 (red dotted lines) obtained using $\omega_{k}=[40: 10: 80]$ when $J=128$ and at noise-levels $\varepsilon=0.02$ (top) and $\varepsilon=0.1$ (bottom). The exact value $\mu_{\mathrm{ex}}$ is shown as a solid black line.
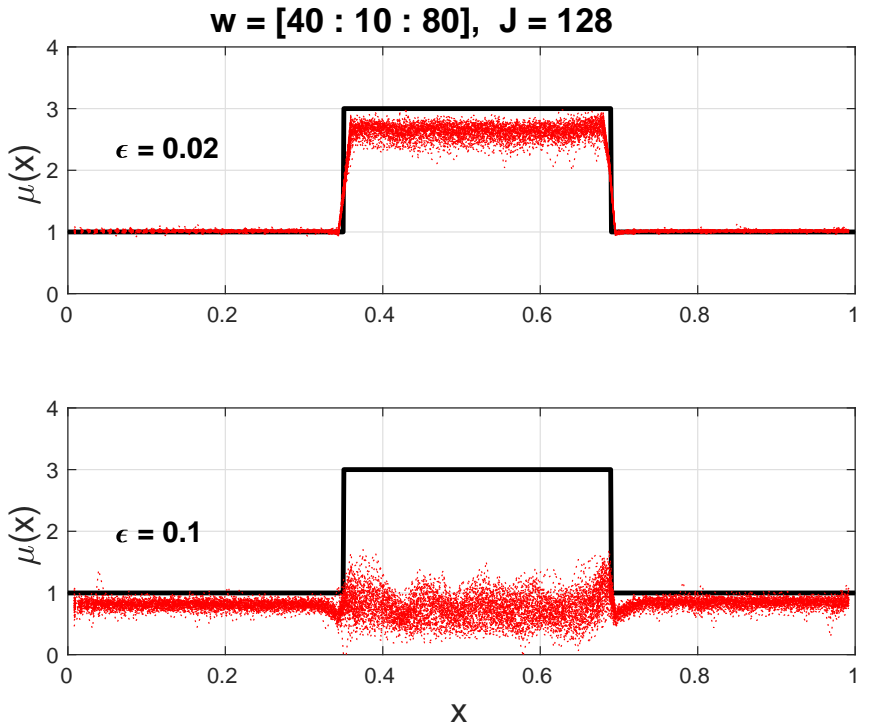

FIG. 2.3. Plot of 100 simulations of calculated $\mu_{\mathrm{LS}}^{*}$ from SFWI-2 (red dotted lines) obtained using $\omega_{k}=[40: 10: 80]$ when $J=128$ and at noise-levels $\varepsilon=0.02$ (top) and $\varepsilon=0.1$ (bottom). The exact value $\mu_{\mathrm{ex}}$ is shown as a solid black line. 

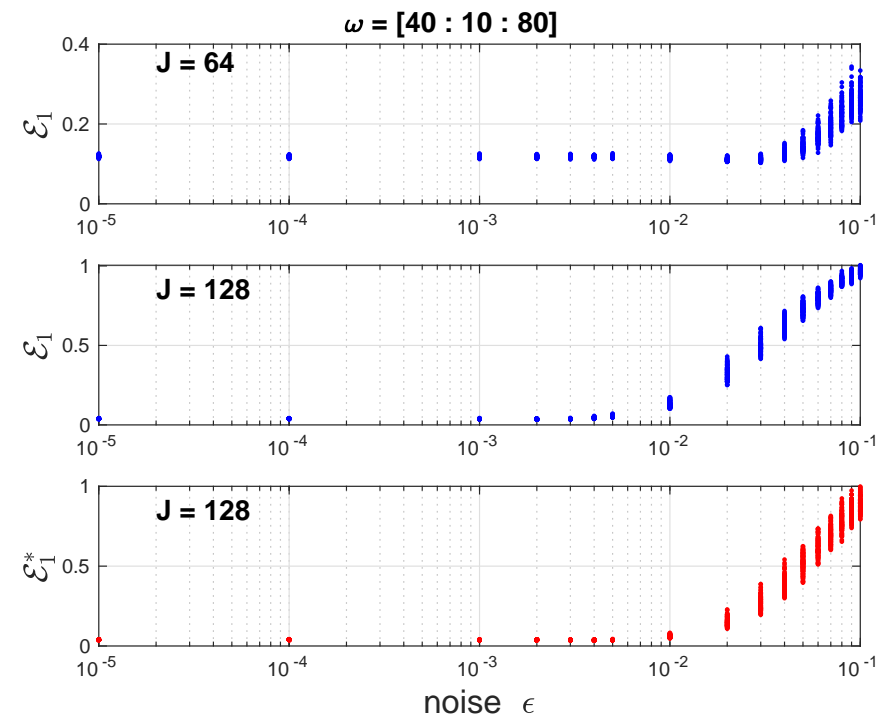

FIG. 2.4. Blue dots: plot of $L^{1}$-error $\mathscr{E}_{1}$ in $\mu_{\text {LS }}$ from SFWI- 1 against noise-level $\varepsilon$ obtained using $\omega_{k}=[40: 10: 80]$ when $J=64$ (top) and $J=128$ (middle). Red dots: plot of $L^{1}$-error $\mathscr{E}_{1}^{*}$ in $\mu_{\mathrm{LS}}^{*}$ from SFWI-2 when $J=128$ (bottom) at the same $\omega_{k}$ (the plot of $\mathscr{E}_{1}^{*}$ when $J=64$ is very similar to the top plot). There are 100 simulations at each value of $\varepsilon$.

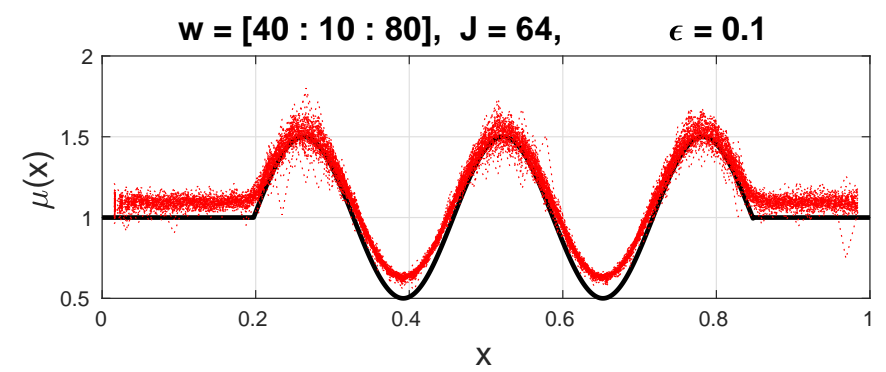

FIG. 2.5. Plot of 100 simulations of calculated $\mu_{\mathrm{LS}}^{*}$ (red dotted lines) obtained using $\omega_{k}=[40: 10: 80]$ in algorithm SFWI-2 when $J=64$ and at noise-level $\varepsilon=0.1$. The exact value $\mu_{\mathrm{ex}}$ is shown as a solid black line. 
The tests indicate that SFWI-2 performs as well as, or better than, SFWI-1 and we conclude this section with SFWI-2 results for more complicated synthetic materials. Figure 2.5 shows a material whose shear modulus varies sinusoidally, and this is robust to noise (when $J=64$ ). But Figure 2.6 illustrates that other examples are less robust to noise.
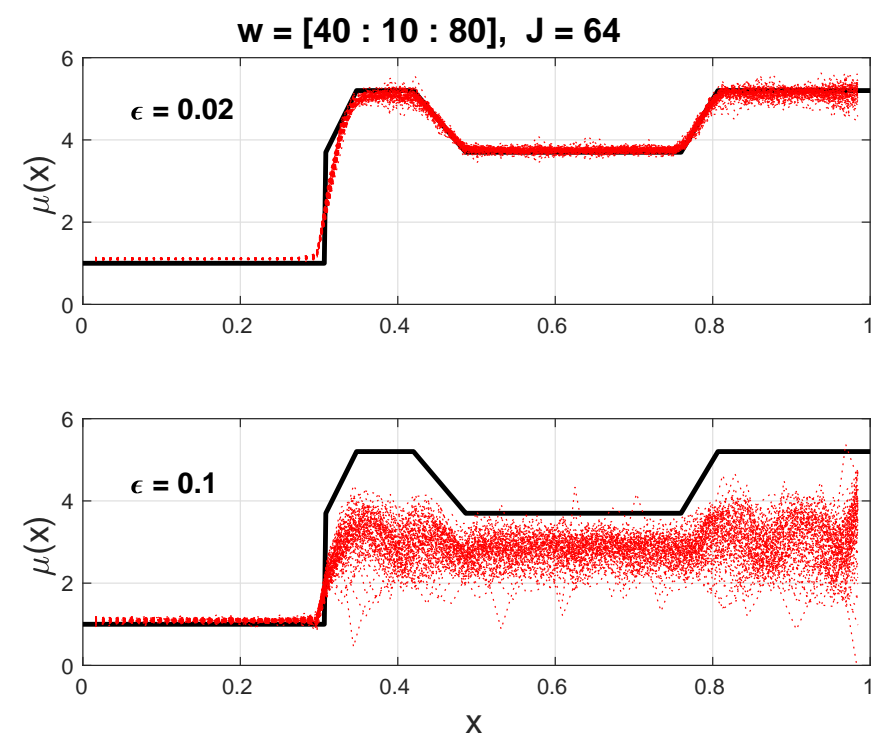

FIG. 2.6. Plot of 100 simulations of calculated $\mu_{\mathrm{LS}}^{*}$ (red dotted lines) obtained using $\omega_{k}=[40: 10: 80]$ in algorithm SFWI-2 when $J=64$ and at noise-levels $\varepsilon=0.02$ (top) and $\varepsilon=0.1$ (bottom). The exact value $\mu_{\text {ex }}$ is shown as a solid black line.

\section{Approximation in 2D and 3D space}

In higher dimensions we use measured values of $\mathbf{u}(\mathbf{x}, t)$ and the nondimensionalized SWE (1.3) to determine the shear modulus. The approach is essentially the same as in 1D: we take a finite volume approximation of (1.3) over each space element (pixels in 2D and voxels in 3D) and replace $\mathbf{u}$ by its Fourier interpolant in time to obtain underdetermined linear systems like (2.9). These are then 'stacked' into a least squares problem like (2.11), and we again use $\mu_{\mathrm{LS}}$ to denote the solution obtained using $M-1$ Fourier components in the higher dimensional analogue of SFWI- 1 and $\mu_{\mathrm{LS}}^{*}$ for the higher dimensional analogue of SFWI-2 (using the two dominant Fourier components). In both cases a similar argument to that used in the 1D case shows that the least squares solution is again real (in perfect arithmetic) and the calculated solutions are real up to rounding error.

We use the same central difference approximations for the spatial derivatives in (1.3) as those for the frequency domain problem described in Davies et al. (2019). We again use a staggered spatial mesh, with the meshpoints for $\mathbf{u}$ being the pixel/voxel midpoints. In 2D the meshpoints for the shear modulus $\mu(\mathbf{x})$ are the midpoints of the interior pixel edges (as shown in Fig. 4.2 of Davies et al. (2019)), and in 3D they are the face centres of the interior voxels. The calculated shear modulus values are then averaged over each pixel/voxel (e.g. the value output for each interior pixel in $2 \mathrm{D}$ is the average of the value at four edge midpoints surrounding that pixel). 
We now present results for 3D experimental displacement measurements from an inhomogeneous gel sample. The results are for a 3D sample, but we also consider them in 2D by ignoring the dependance of (1.3) on $x_{3}$.

\subsection{D experimental test data}

The two variants of the algorithm are tested on data obtained from an inhomogeneous gel 'phantom' matrix which contains four circular cylindrical gel inclusions of different stiffness and diameter $12 \mathrm{~mm}$. The shear modulus of the background matrix and cylinders have been determined by torsional rheometry in Papazoglou et al. (2012) and are listed in Table 1. These data are also reported in Tzschätzsch et al. (2016).

\begin{tabular}{c|c|c|c|c}
\hline Matrix & incl. 1 & incl. 2 & incl. 3 & incl. 4 \\
\hline 10.8 & 43.3 & 5.2 & 6.0 & 16.3 \\
\hline
\end{tabular}

Table 1. Shear modulus $\mu$ (kPa) for the matrix and four inclusions, determined by torsional rheometry Papazoglou et al. (2012).

MRI displacement measurements are taken with underlying oscillation frequency $f \mathrm{~Hz}$ for $f=$ $30: 10: 100$ using $M=8$, and the voxels are cubes of side $1.5 \mathrm{~mm}$. The displacements are measured on a spatial grid of size $J_{1} \times J_{2} \times J_{3}$ with $J_{1}=128, J_{2}=80$ and $J_{3}=25$, and we note that some of the measurements are taken outwith the gel sample in the $x_{1} x_{2}$ plane - at these points the recorded measurement is just noise. The measurements all lie within the gel sample in the $x_{3}$ plane and form a layer 25 voxels deep, with 23 interior voxels. All our plots show the variation of the calculated shear modulus with $x_{1}$ and $x_{2}$ in a single representative slice in the $x_{3}$ direction, typically slice 9 out of the 23 interior slices (i.e. slice 10 out of 25 in total). We plot the $x_{2}$ variable downwards to aid comparison with Tzschätzsch et al. (2016), and the order of inclusions is 1: top left; 2: top right; 3: bottom left; 4 : bottom right.

The figures in Fig. 4 of Tzschätzsch et al. (2016) and below indicate that the gel phantom occupies about $64 \%$ of the measured area in the $x_{1} x_{2}$ plane, and so the mean value of the shear modulus over the total measured volume is very roughly $7 \mathbf{~ k P a}$. In practice MRI measurements are highly smoothed before being used to calculate the shear modulus, and the necessity of this is illustrated by Figure 3.1 which shows the 2D slice of $\mu_{\mathrm{LS}}$ calculated using the 3D analogue of SFWI-1 obtained from raw data with no smoothing. The left-hand plot is calculated using all eight measurements $(f=30: 10: 100 \mathrm{~Hz})$ and the right-hand plot uses the four measurements with highest oscillation frequency $(f=70: 10: 100$ $\mathrm{Hz}$ ). Whilst the plots clearly show the gel sample and inclusions 1 (top left) and 4 (bottom right), both calculated values are completely wrong (the average shear modulus over the total measured volume are 0.37 and 0.57 respectively, an order of magnitude away from the actual value of about 7), and the second is about 50\% higher than the first. Note also that the shear modulus of these two inclusions is higher than that of the underlying sample, but the figure shows them as darker (lower). This is likely to be because a stiffer material (i.e. higher $\mu$ ) has a shorter relaxation time, which results in increased signal noise and hence a worse approximation.

The results in Figure 3.1 are surprising in that the inclusions are visible even without any data smoothing, although the calculated shear modulus is completely wrong. They also illustrate that published results in the MRE literature which use plots alone have the potential to be very misleading. 

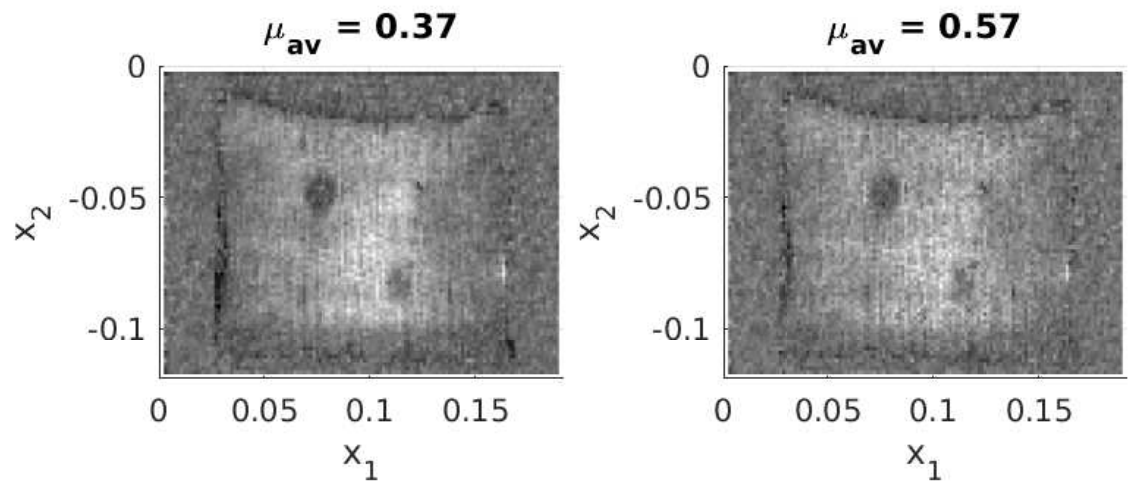

FIG. 3.1. Plots of calculated $\mu_{\mathrm{LS}}$ from the unsmoothed 3D dataset obtained using SFWI-1, shown for interior voxel slice 9 out of 23 in the $x_{3}$ direction. The title indicates the average value of $\mu_{\mathrm{LS}}$ over the full 3D volume (which includes measurements from outwith the gel sample; see text for details). LEFT: the stacked matrix and vector uses all eight measurements $(f=30: 10: 100$ $\mathrm{Hz})$; RigHT: the stacked matrix and vector use the four measurements with highest oscillation frequency $(f=70: 10: 100 \mathrm{~Hz})$.

One of the simplest ways to smooth measured data is to filter out 'sawtooth' components which oscillate as \pm 1 between neighbouring cells (intervals, pixels or voxels, depending on the space dimension $d$ ). The formula for this is

$$
\mathbf{u}_{\text {new }}=\left(1+C[d] \sum_{\alpha=1}^{d} \delta_{\alpha \alpha}\right) \mathbf{u}
$$

where $\delta_{\alpha \alpha}$ is the standard second central difference operator (i.e. $\delta_{11} \phi_{j, k, \ell}=\phi_{j+1, k, \ell}-2 \phi_{j, k, \ell}+\phi_{j-1, k, \ell}$, etc.) and $C[d]$ is a dimension-dependent coefficient chosen to eliminate modes of the form $(-1)^{j+k+\ell}$. It is straightforward to verify that $C[d]=1 /(4 d)$. Figure 3.2 shows the result of applying this unsophisticated 'Laplacian' smoothing to the measured displacement values u before using algorithm SFWI-1 with the four highest frequency oscillations $(f=70: 10: 100 \mathrm{~Hz})$ to obtain $\mu_{\mathrm{LS}}$. Applying it once (left plot) increases the average shear modulus from 0.57 to 1.0, and applying it twice (right plot) increases it again to 1.6. These values are still significantly lower than the actual value, but are now within an order of magnitude. The gel phantom is much more clearly delineated than in Figure 3.1, but its features are smeared out. Applying it for a third time results in a much more smeared image (not shown), and the average shear modulus is now 2.2 .

\subsection{3 experimental test data used in $2 D$ calculations}

The gel phantom sample is axially homogeneous (it does not vary in the $x_{3}$ direction), and we now investigate the effect on the shear modulus of calculating it independently from each 2D slice of data. To do this we ignore the third equation of (1.3) and all space derivatives with respect to $x_{3}$. The main advantage of this approach is that the least squares problems are smaller and significantly faster to solve, but as observed in Davies et al. (2019), measurements in higher dimensions provide a much richer data source to solve from. 

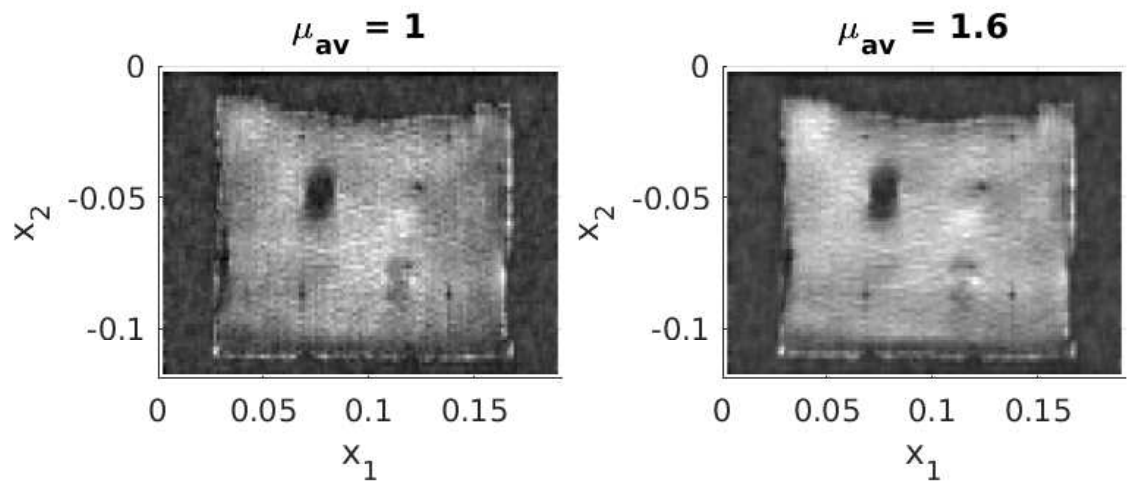

FIG. 3.2. Plots of calculated $\mu_{\mathrm{LS}}$ obtained using SFWI-1 on the 3D dataset after it has been Laplacian smoothed (once for the left-hand plot and twice for the right-hand plot), shown for interior voxel slice 9 out of 23 in the $x_{3}$ direction, with the results obtained from the four measurements with highest oscillation frequency $(f=70: 10: 100 \mathrm{~Hz})$. The title indicates the average value of $\mu_{\mathrm{LS}}$ over the full $3 \mathrm{D}$ volume.
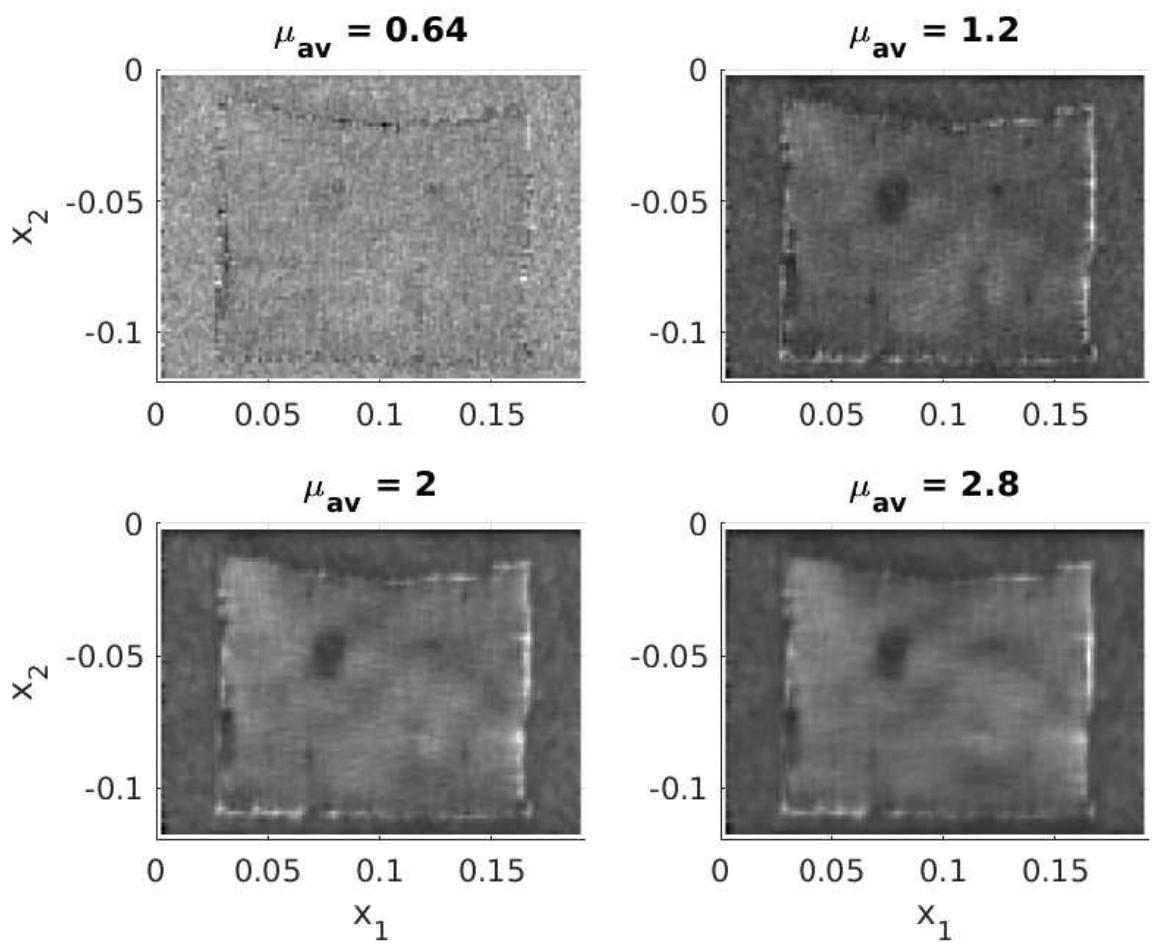

FIG. 3.3. Plots of $\mu_{\text {LS }}$ calculated from the 2D analogue of SFWI- 1 for slice 10 obtained from the four measurements with highest oscillation frequency $(f=70: 10: 100 \mathrm{~Hz})$. The title indicates the average value of $\mu_{\mathrm{LS}}$ over the $2 \mathrm{D}$ area given in $\mathrm{kPa}$. TOP LEFT: unsmoothed measurement data; TOP RIGHT: measurement data has been 2D-Laplacian smoothed once; BOTTOM LEFT: measurement data has been 2D-Laplacian smoothed twice; BотTOM RIGHT: measurement data has been 2D-Laplacian smoothed three times. 
The results using the 2D analogue of SFWI-1 are similar to those in 3D, but with slightly higher average values for the shear modulus (the average is now taken over one slice, rather than the entire volume). The results do not vary much between slices, but tend to be slightly better for those towards the far end (higher index), but for consistency we again focus on slice 10 (out of 25). For example, when all eight measurements $(f=30: 10: 100 \mathrm{~Hz})$ are used in the stacked matrix and vector, then the average shear modulus for unsmoothed data is $0.4 \mathrm{kPa}$, and for data which has been (2D) Laplacian smoothed one, two or three times it is $0.76,1.3$, and 2.0 respectively. As in 3D the results appear better when the lower frequencies are not included, and those using oscillation frequency ( $f=70: 10: 100$ $\mathrm{Hz}$ ) are shown in Figure 3.3. One possible reason for the slight improvement in the 2D results over those from the full 3D data set is that the mechanical oscillations (and hence change in displacement) may be predominantly in the $x_{1} x_{2}$ plane.

We now compare these results with the 2D analogue of SFWI-2 in which only the dominant Fourier components are used. As shown in Fig. 3.4, the gel sample appears more clearly delineated in this case (there is less dominant frequency noise in the surrounding air), but the overall average values are slightly lower than for the SFWI-1 case.
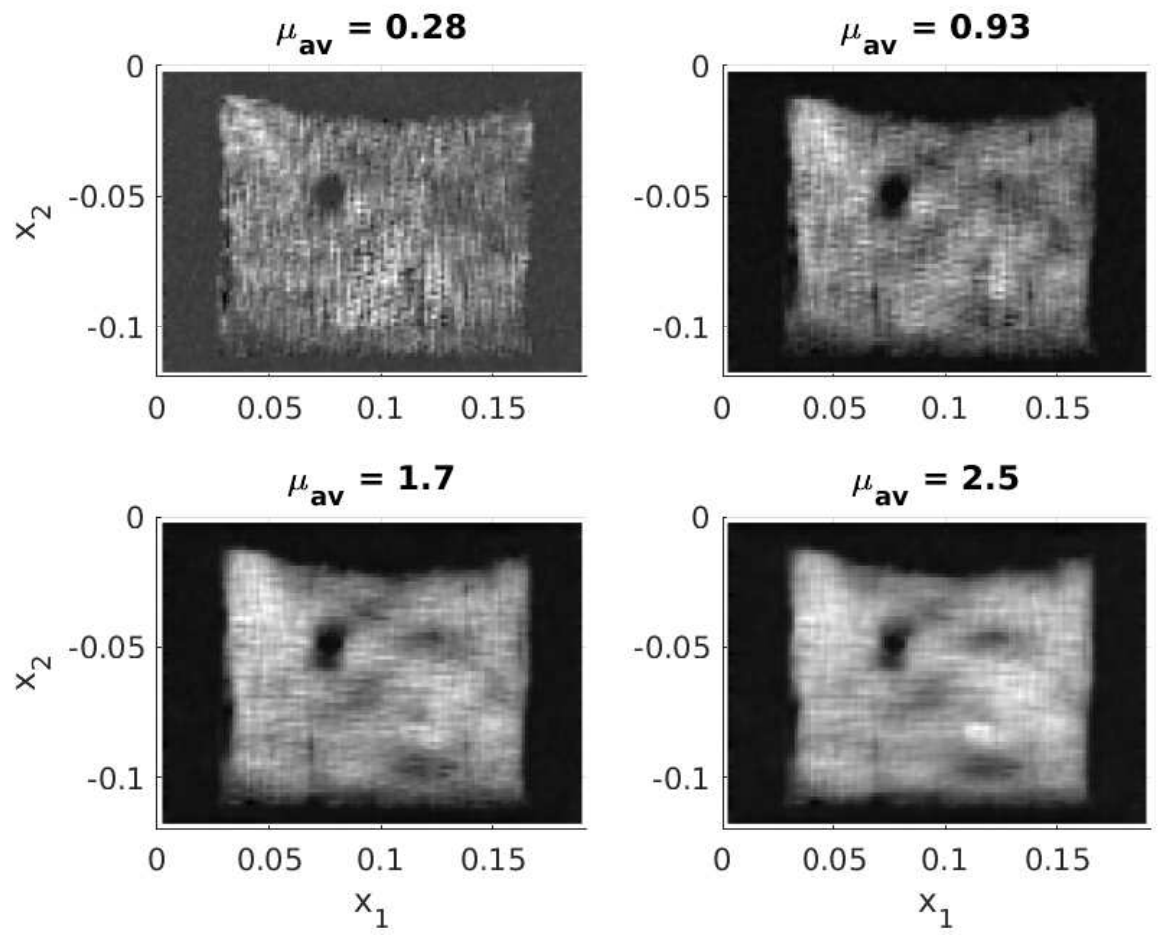

FIG. 3.4. Plots of $\mu_{\mathrm{LS}}^{*}$ calculated from the 2D analogue of SFWI-2 for slice 10 (out of 25) obtained from the four measurements with highest oscillation frequency $(f=70: 10: 100 \mathrm{~Hz})$. The title indicates the average value of $\mu_{\mathrm{LS}}^{*}$ over the 2D area given in $\mathrm{kPa}$. TOP LEFT: unsmoothed measurement data; TOP RIGHT: measurement data has been 2D-Laplacian smoothed once; BOTTOM LEFT: measurement data has been 2D-Laplacian smoothed twice; BotTOM RIGHT: measurement data has been 2D-Laplacian smoothed three times. 
Our 1D simulations indicate that MRE results are more resilient in the presence of noise if the space mesh is coarsened and Fig. 3.5 shows the same calculations as for Fig. 3.4 but now obtained using alternate data points (i.e. $\Delta x_{1}$ and $\Delta x_{2}$ are doubled). This does yield much higher (i.e. more realistic) average values of $\mu_{\mathrm{LS}}^{*}$, although the reduced resolution makes it harder to see the gel inclusions.
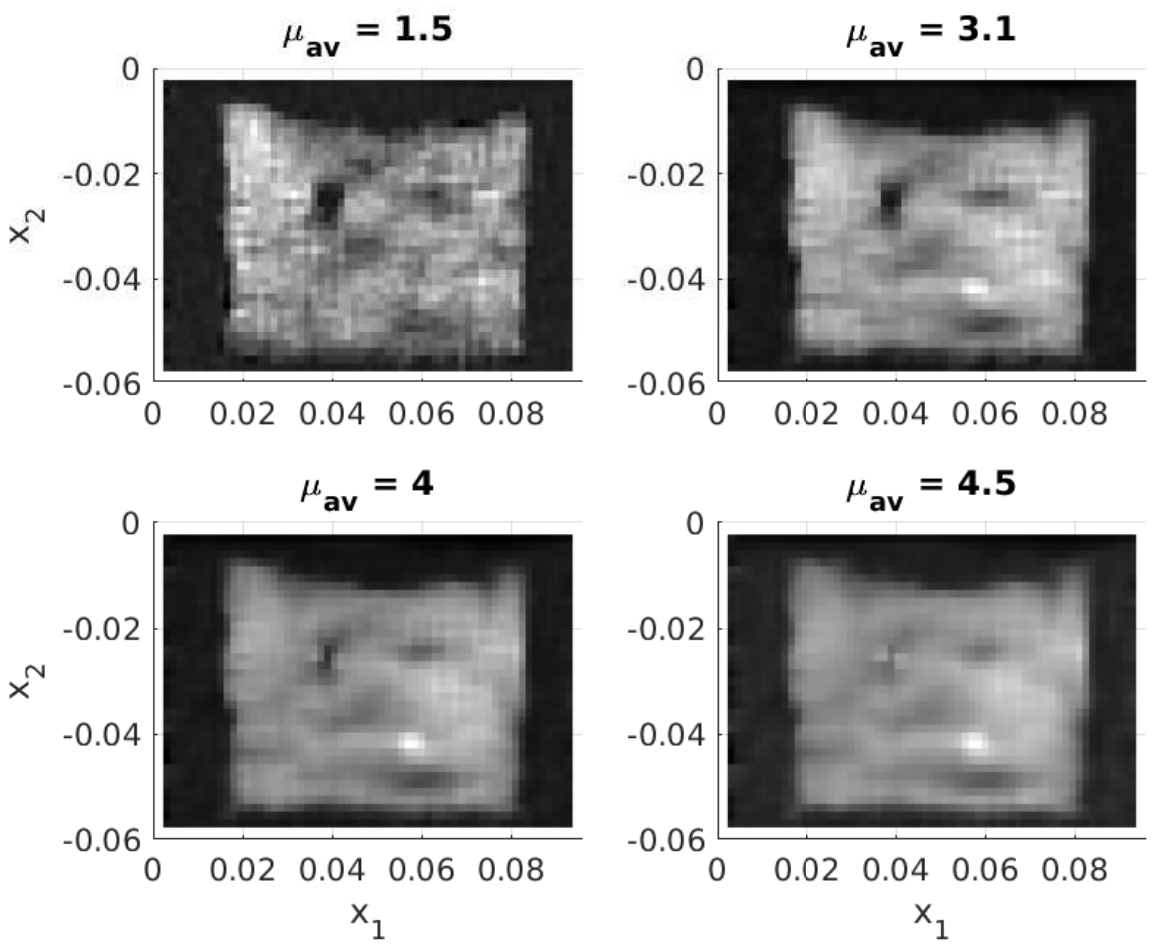

FIG. 3.5. Plots of $\mu_{\mathrm{LS}}^{*}$ calculated from the 2D analogue of SFWI- 2 for slice 10 obtained from the four measurements with highest oscillation frequency $(f=70: 10: 100 \mathrm{~Hz})$. This is the same data sample as used for Fig. 3.4 but the results here only use every other data point (i.e. $\Delta x_{1}$ and $\Delta x_{2}$ are doubled). The title indicates the average value of $\mu_{\mathrm{LS}}^{*}$ over the $2 \mathrm{D}$ area given in $\mathrm{kPa}$. TOP LEFT: unsmoothed measurement data; TOP RIGHT: measurement data has been 2D-Laplacian smoothed once; BOTTOM LEFT: measurement data has been 2D-Laplacian smoothed twice; BотTом RIGHT: measurement data has been 2D-Laplacian smoothed three times.

These $2 \mathrm{D}$ and $3 \mathrm{D}$ results confirm that noise is a very serious issue for MRE, and that (much more) sophisticated smoothing of the displacement measurements will be necessary to obtain reasonable results. We now investigate the effect of noise in more detail.

\section{The effect of noise}

Inverse problems arising in image deblurring and denoising and other applications areas are often modelled by ill-posed operator equations which are then discretized to give large-scale linear systems like

$$
B \mathbf{z}=\mathbf{c},
$$


where $B \in \mathbb{R}^{n \times n}$ (modelling the process) and $\mathbf{c} \in \mathbb{R}^{n}$ (modelling the output) are known, and the aim is compute an accurate approximation of the 'input' $\mathbf{z} \in \mathbb{R}^{n}$. As noted in Gazzola et al. (2015) (see also Chung \& Gazzola (2019) and Gazzola \& Sabaté Landman (2019)), a typical scenario is for the right-hand side $\mathbf{c}$ to be affected by noise, but the ill-conditioned matrix $B$ is essentially noise-free with singular values that decay extremely rapidly. If its singular value decomposition (SVD) is $B=U \Sigma V^{T}$ for orthogonal $U, V$ and diagonal $\Sigma=\operatorname{diag}\left(\sigma_{1}, \ldots, \sigma_{n}\right)$, then the solution of (4.1) can be written as

$$
\mathbf{z}=\sum_{j=1}^{n} \frac{\mathbf{u}_{j}^{T} \mathbf{c}}{\sigma_{j}} \mathbf{v}_{j}
$$

where $\mathbf{u}_{j}$ (respectively $\mathbf{v}_{j}$ ) is the $j$ th column of $U$ (respectively $V$ ). If the noise in the right-hand side of (4.1) is $\mathbf{e}$ (i.e. $\mathbf{c}=\mathbf{c}_{\mathrm{ex}}+\mathbf{e}$ ) then the solution error in the direction $\mathbf{v}_{j}$ is $\left|\mathbf{u}_{j}^{T} \mathbf{e}\right| / \sigma_{j}$, which can be very large for the many values of $j$ for which the singular value $\sigma_{j}$ is very small. As discussed in Gazzola et al. (2015), two well-established techniques to overcome this are to (i) regularize the problem, instead considering

$$
\min _{\mathbf{z} \in \mathbb{R}^{n}}\left\{\|B \mathbf{z}-\mathbf{c}\|^{2}+\lambda^{2}\|L \mathbf{z}\|^{2}\right\},
$$

where $\lambda>0$ is a regularization parameter and $L$ is a regularization matrix, and (ii) use a Krylov iterative method for (4.2), stopping after $k \ll n$ iterations.

The MRE problem (2.11) (in whatever space dimension) has the form (4.1) with $B=\tilde{A}^{H} \tilde{A}$ and $\mathbf{c}=\tilde{A}^{H} \tilde{\mathbf{b}}$, which as we have previously seen are both real, and $\mathbf{z}$ is either $\mu_{\mathrm{LS}}$ or $\mu_{\mathrm{LS}}^{*}$, depending on whether the underlying algorithm is SFWI-1 or SFWI-2. However a crucial difference is that the error in $B$ due to noise is significantly larger than that in $\mathbf{c}$ (in the notation of Section 2 it is $\varepsilon^{2} / \Delta x^{2}$ compared to $\varepsilon^{2}$ ). Denoting the matrix and right-hand side vector with no noise as $B_{\mathrm{ex}}$ and $\mathbf{c}_{\mathrm{ex}}$ and the errors due to noise as $\delta B$ and $\delta \mathbf{c}$ respectively, then $B_{\mathrm{ex}}$ and $\delta B$ have the same sparsity pattern and the 'noisy' solution satisfies

$$
\left(B_{\mathrm{ex}}+\delta B\right) \mathbf{z}=\mathbf{c}_{\mathrm{ex}}+\delta \mathbf{c} \approx \mathbf{c}_{\mathrm{ex}} .
$$

As $\Delta x$ is reduced the size of $\delta B$ increases, which is why the approximate solution size is reduced more for a finer mesh. We now look at the behaviour of the singular values of $B$ in one and two space dimensions.

\subsection{Singular values of $B=\tilde{A}^{H} \tilde{A}$}

Fig. 4.1 shows the behaviour of the singular values of the MRE matrix $B$ for the synthetic $1 \mathrm{D}$ algorithm SFWI-1 calculation illustrated in Figs 2.1-2.2 for different values of $\Delta x=1 / J$. Added noise clearly affects the later singular values more as the mesh is refined, but note also that the singular values behave quite differently to those for image deblurring problems as considered in e.g. Gazzola et al. (2015), where $\sigma_{j}$ decays very rapidly with $j$. The SFWI-2 version is shown in Fig. 4.2, and in this case the singular values are less affected by noise, despite the two algorithms giving very similar results.

The first 750 singular values for the 2D SFWI- 2 calculation considered in the previous section (slice 10 using the four measurements with highest oscillation frequency $f=70: 10: 100 \mathrm{~Hz}$ ) are shown in the top plot of Fig. 4.3 for data which has been (2D) Laplacian smoothed $k$ times for $k=0: 3$ (the MRE results for this case are given in Fig. 3.4). The bottom plot is obtained by only considering every other data point (i.e. $\Delta x_{1}$ and $\Delta x_{2}$ are doubled), and its singular values decay more rapidly (the MRE results for this case are given in Fig. 3.5). 

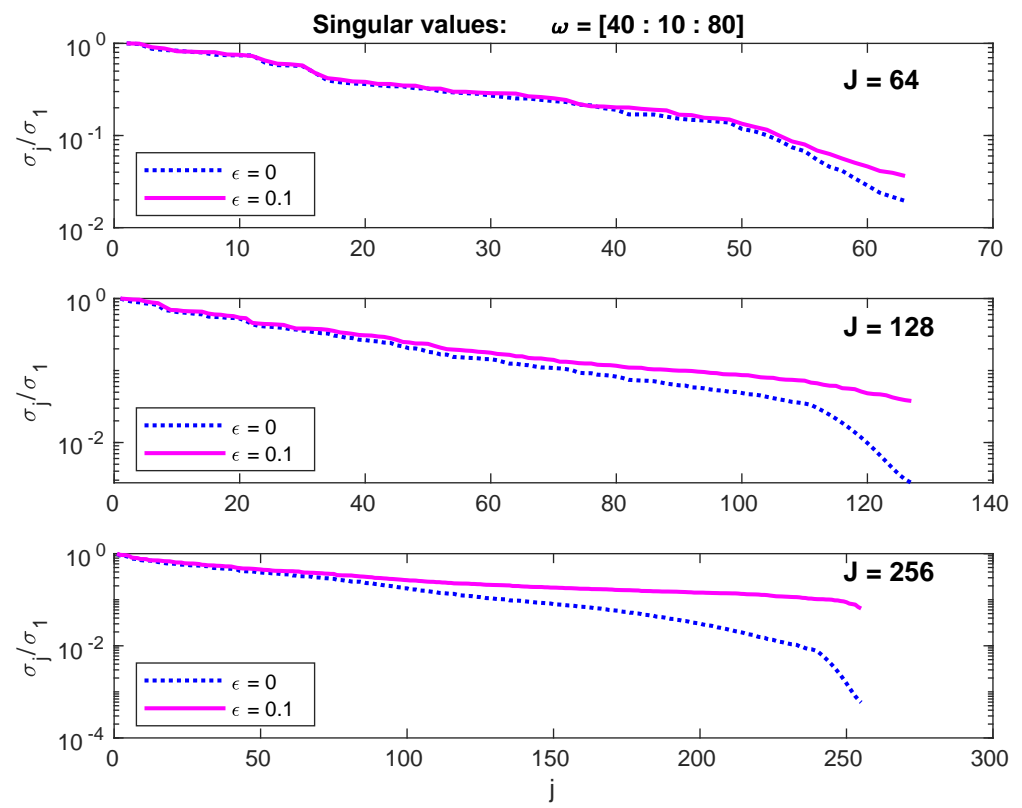

FIG. 4.1. Plots of the scaled singular value $\sigma_{j} / \sigma_{1}$ of $B=\tilde{A}^{H} \tilde{A}$ shown for the algorithm SFWI-1 version of the $1 \mathrm{D}$ synthetic calculation at the start of Section 2.4. Each plot shows the scaled singular values with no added noise (blue dotted) and when $\varepsilon=0.1$ (i.e. $10 \%$ added noise).

\subsection{Conjugate gradient iteration of (4.2) in $1 D$ and $2 D$ space}

The regularized problem (4.2) for MRE is equivalent to the linear system

$$
\left(\tilde{A}^{H} \tilde{A}+\lambda^{2} L^{T} L\right) \mathbf{z}=\tilde{A}^{H} \tilde{\mathbf{b}}
$$

and we investigate the effect of regularization when $L$ is the central finite difference approximate Laplacian operator (i.e. in $1 \mathrm{D}$ space $\left.L v_{j}=\left(v_{j-1}-2 v_{j}+v_{j+1}\right) / \Delta x^{2}\right)$. Including this term makes the calculated solution smoother, with more smoothness as $\lambda$ is increased, but unfortunately it appears to have no effect on whether or not the 1D algorithm works, with the SFWI- 1 results for $J=64$ and $J=128$ at $10 \%$ noise looking like a smoother version of the bottom plots in Figs 2.1-2.2. Using a small number of iterations of the conjugate gradient (CG) method, described in e.g. Golub \& Loan (1996) for (4.3) also appears to have no discernible effect on the $1 \mathrm{D}$ algorithm, whatever value of $\lambda$ is chosen, in contrast to the image deblurring applications considered in Gazzola et al. (2015). Starting with the initial value $\mathbf{z}=\mathbf{0}$ and stepping through the CG iterates gives an approximate solution which gradually increases and then shows no further change. As before, the algorithm works well on a coarse mesh or for low noise levels, but not when $J=128$ and $\varepsilon=0.1$. This "end result" for the SFWI-1 version of (2.1) with $\omega=[40: 10: 80]$ is shown in Figs 4.4-4.5. Note that the square of the second central difference operator in each case is multiplied by $\lambda^{2} / \Delta x^{4}$, and this constant is very large even for relatively small values of $\lambda$. 

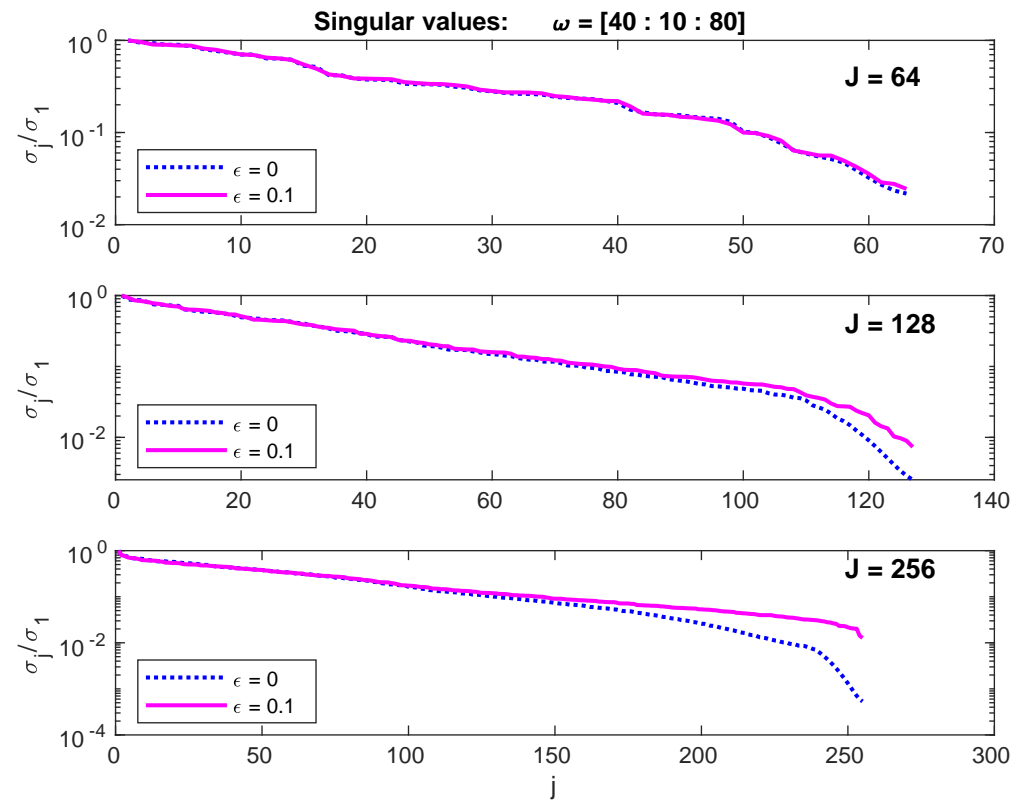

FIG. 4.2. Plots of the scaled singular value $\sigma_{j} / \sigma_{1}$ of $B=\tilde{A}^{H} \tilde{A}$ shown for the algorithm SFWI-2 version of the 1D synthetic calculation at the start of Section 2.4. Each plot shows the scaled singular values with no added noise (blue dotted) and when $\varepsilon=0.1$ (i.e. $10 \%$ added noise).

Because the value of $\lambda$ appears to make little difference we only consider the $\lambda=0$ case when calculating CG iterates for the 2D "data slice" problem considered in Sec. 3.2. As in 1D, stepping through the CG iterates (from the initial value $\mathbf{z}=\mathbf{0}$ ) gives a gradually increasing approximate solution, and although its actual value is very inaccurate, the inhomogeneities in the gel are more apparent when watching a movie of the iterates than in a static solution plot. A snapshot for the SFWI-2 version of the calculation for data slice 10 (out of 25) obtained from the four measurements with highest oscillation frequency $(f=70: 10: 100 \mathrm{~Hz})$ which have been Laplacian smoothed twice are given in Fig. 4.6: the first three plots show different CG iterates, and the full solution of (4.3) with $\lambda=0$ is shown in the bottom right (this is the same plot as the bottom left of 3.4).

\section{Discussion and conclusions}

We have described and analysed two new methods for time-dependent MRE: algorithms SFWI-1 and SFWI-2. Both of them automatically give a real value of the computed shear modulus (a real quantity), which is an important property not shared by methods which use a single dominant DFT component. There is little difference in the algorithms' performance in the presence of 'random' errors, but we anticipate that the SFWI-2 version might be more useful in practical situations in which the experimental equipment introduces a systematic low-frequency vibrational noise compared to the mechanical forcing frequency $f$. 

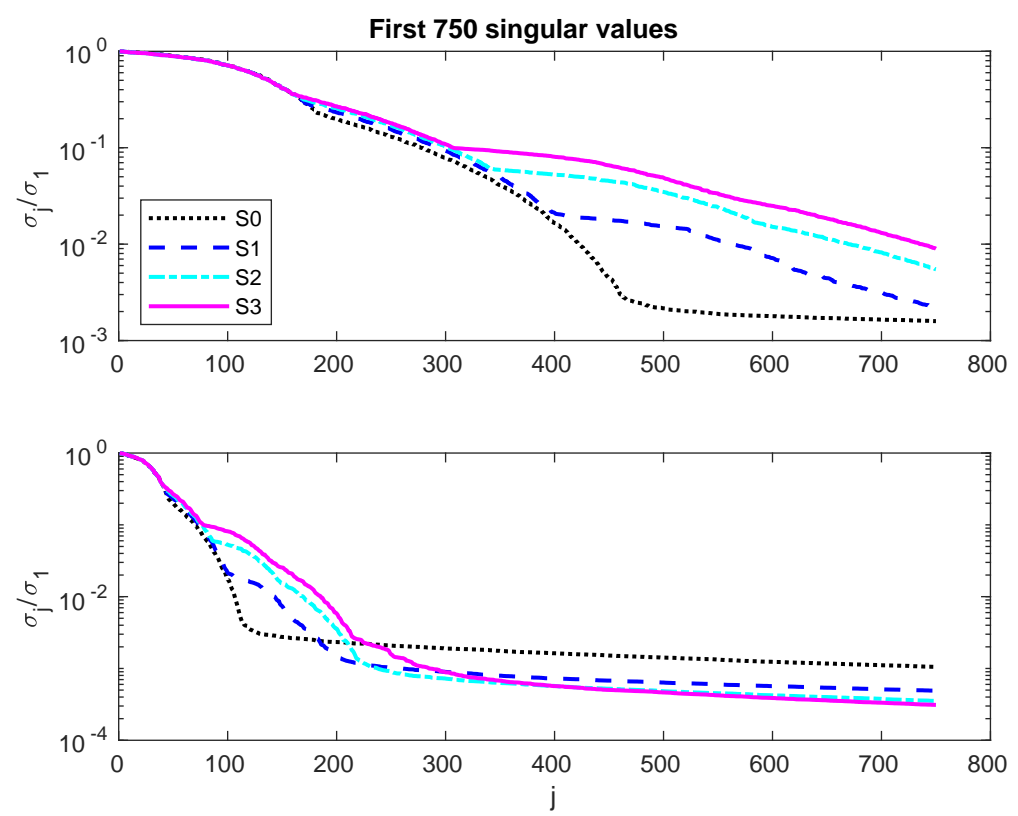

FIG. 4.3. The first 750 scaled singular values $\sigma_{j} / \sigma_{1}$ of $B=\tilde{A}^{H} \tilde{A}$ for the $2 \mathrm{D}$ data slice 10 obtained using algorithm SFWI-2 for the four measurements with highest oscillation frequency $(f=70: 10: 100 \mathrm{~Hz})$. The displacement data points are (2D) Laplacian smoothed $k$ times (labelled as $S k$ in the caption) for $k=0: 4$. Top: uses the full $2 \mathrm{D}$ data sample with $N_{1}=128$ and $N_{2}=80$; Воттом: uses every other data point (thus doubling $\Delta x_{1}$ and $\Delta x_{2}$ and now $N_{1}=64$ and $N_{2}=40$ ).
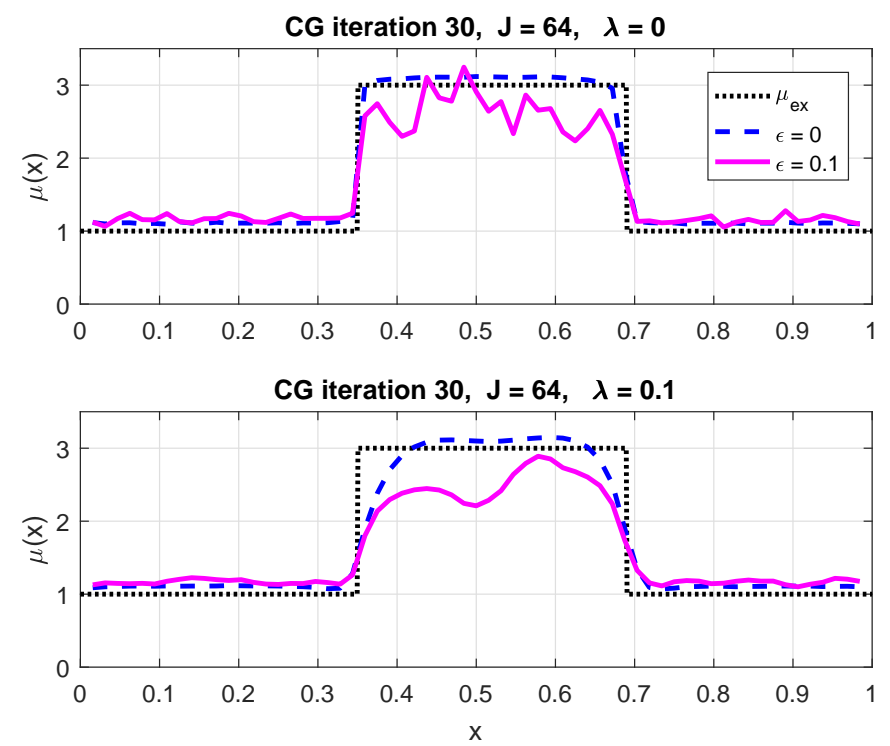

FIG. 4.4. Plot of calculated $\mu_{\mathrm{LS}}$ from CG iteration 30 of (4.3) when $J=64$ and at noise-levels $\varepsilon=0$ (blue dash) and $\varepsilon=0.1$ (maroon solid). The exact value $\mu_{\mathrm{ex}}$ is shown as a black dotted line. Tор PLOT: $\lambda=0$; ВотTом PLOT: $\lambda=0.1$. 

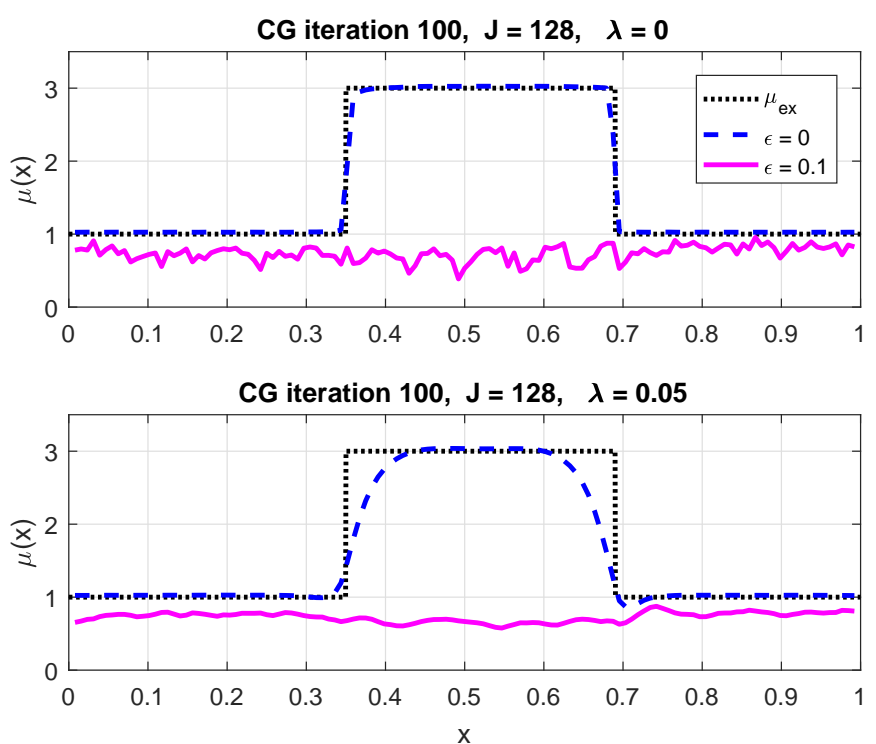

FIG. 4.5. Plot of calculated $\mu_{\mathrm{LS}}$ from CG iteration 100 of (4.3) when $J=128$ and at noise-levels $\varepsilon=0$ (blue dash) and $\varepsilon=0.1$ (maroon solid). The exact value $\mu_{\mathrm{ex}}$ is shown as a black dotted line. Tор PLOT: $\lambda=0$; Воттом PLOT: $\lambda=0.05$.

Experimental tests indicate that both algorithms work very well at low values of noise, but the error in the computed shear modulus grows quickly with increasing noise. The solution error caused by a specific noise level depends on the interval/pixel/voxel size used for the displacement, and when there is a large solution error then it corresponds to the computed shear modulus value being far too small (this is well-known in MRE, as noted in e.g. Arunachalam et al. (2017)). The figures in Sections 3 and 4 indicate that solution plots for the gel phantom typically indicate the position of the inclusions even when the computed shear modulus is very inaccurate. The reason for this is not clear, but it means that MRE results which are given solely as pictures (i.e. without any numerical values) should be treated with caution. Note that the computed values obtained from the two stiffest inclusions are lower than for the ambient material, when they should actually be higher. This is likely to be because the underlying stiffer material has a shorter MRI relaxation time, giving increased measurement noise, and because the approximation is so sensitive to noise larger than some threshhold value this results in a far worse (lower) approximation in these inclusions.

Of course a real material such as tissue or the gel phantom will not be purely elastic, and so it cannot be modelled exactly by an equation like (1.1) or (1.3). This means that there will inevitably some discrepancy between the material's shear modulus and the equations that are being used to calculate it, and this will be a source of error. However the experimental results for the gel phantom (in both 2D and 3D) behave very similarly to the results for the 1D 'toy' problem considered in Section 2, and in this case the "data measurements" accurately solve the 1D equation (2.1), with synthetic noise added in afterwards. This leads us to conjecture that using a purely elastic model is not the main cause of large solution errors in the presence of noise. We are currently investigating solution algorithms for viscous models like (2.13) and its higher dimensional analogue.

Another aspect that we have not considered is how the displacement data can be reliably filtered to remove the gradient term from (1.1) in order to obtain (1.2). This is not obvious, especially since div $\mathbf{u}$ 

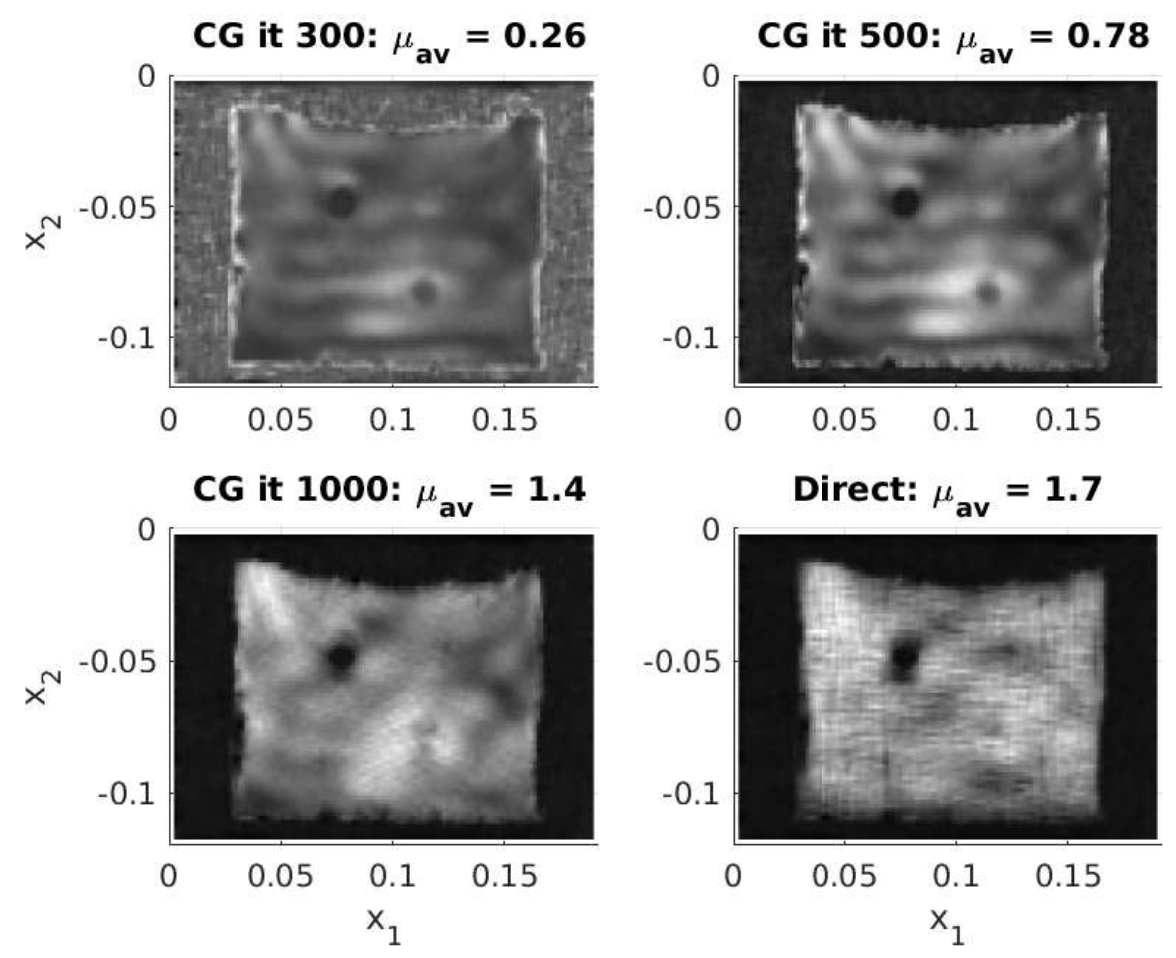

FIG. 4.6. Plots of $\mathbf{z}=\mu_{\mathrm{LS}}^{*}$ calculated from (4.3) for the 2D analogue of SFWI- 2 for dataslice 10 obtained from the four measurements with highest oscillation frequency $(f=70: 10: 100 \mathrm{~Hz})$ which have been Laplacian smoothed twice. The title gives the average value of $\mu_{\mathrm{LS}}^{*}$ over the 2D area, in kPa. TOP LEFT: CG iteration 300; TOP RIGHT: CG iteration 500; BOtTOM LEFT: CG iteration 1000; BOTTOM RIGHT: full solution of (4.3).

can only be obtained as a fairly crude approximation from the displacement data, and we are currently investigating ways of dealing with the term $\lambda u_{\beta, \beta}$ in (1.1). However, the similarity of solution behaviour in higher space dimensions to the $1 \mathrm{D}$ case leads us to believe that ignoring the divergence term in (1.1) is also not the main cause of error.

The results in Section 4 indicate that MRE is a different type of inverse problem to those of image processing considered by e.g. Gazzola et al. (2015), and that regularization or (CG) iteration techniques do not improve the MRE solution. But looking at a movie of the CG iterates can indicate areas of interest in the sample, and we would recommend this as a useful tool for practitioners. Our main recommendations are that displacement data measurements will need to be smoothed in order to obtain any sort of solution accuracy (and more smoothing is needed than the simple Laplacian smoothers considered here); and that the analogues of $A$ and $\mathbf{b}$ in (2.9) should contain the complex conjugates of all Fourier components that they use (this is achieved by both SFWI-1 and SFWI-2). At first sight these two suggestions may appear hard to reconcile - if smoothing involves projection onto a spatial subspace then the optimal projection may be different at different time-steps (or Fourier components). However, the SFWI-2 version of the algorithm can always be implemented by applying smoothing to the dominant Fourier component of the displacement, and using this smoothed displacement and its complex conjugate in the $A$ and $\mathbf{b}$ which are then stacked in (2.10). 


\section{Acknowledgment}

This work was supported by the German Research Foundation GRK2260 BIOQIC.

\section{REFERENCES}

Arunachalam, S. P., Rossman, P. J., Arani, A., Lake, D. S., Glaser, K. J., Trzasko, J. D., Manduca, A., McGee, K. P., Ehman, R. L. \& Araoz, P. A. (2017) Quantitative 3D magnetic resonance elastography: Comparison with dynamic mechanical analysis. Magn. Reson. Med., 77(3), 1184-1192.

Barnhill, E., Davies, P. J., Ariyurek, C., Fehlner, A., Braun, J. \& Sack, I. (2018) Heterogeneous multifrequency direct inversion (HMDI) for magnetic resonance elastography with application to a clinical brain exam. Med. Image Anal., 46, 180-188.

Chung, J. \& Gazzola, S. (2019) Flexible Krylov methods for $\ell_{p}$ regularization. SIAM J on Scientific Computing, 41(5), S149-S171.

Davies, P. J., Barnhill, E. \& Sack, I. (2019) The MRE Inverse Problem for the Elastic Shear Modulus. SIAM J. Appl. Math., 79(4), 1367-1388.

Dittmann, F., Hirsch, S., Tzschätzsch, H., Guo, J., Braun, J. \& Sack, I. (2016) In vivo wideband multifrequency MR elastography of the human brain and liver. Magn. Reson. Med., 76, 1116-26.

Doyley, M. M. (2012) Model-based elastography: a survey of approaches to the inverse elasticity problem. Phys. Med. Biol, 57, R35-R73.

Gazzola, S., Novati, P. \& Russo, M. R. (2015) On Krylov projection methods and Tikhonov regularization. Electronic Transactions on Numerical Analysis, 44, 83-123.

Gazzola, S. \& Sabaté Landman, M. (2019) Flexible GMRES for total variation regularization. BIT Numerical Mathematics, 59(3), 721-746.

Golub, G. H. \& Loan, C. F. V. (1996) Matrix Computations. Johns Hopkins University Press, Baltimore, third edition.

Manduca, A., Oliphant, T. E., Dresner, M. A., Mahowald, J. L., Kruse, S. A., Amromin, E., Felmlee, J. P., Greenleaf, J. F. \& Ehman, R. L. (2001) Magnetic resonance elastography: non-invasive mapping of tissue elasticity. Med. Image Anal., 5, 237-254.

McLaughlin, J. R., Zhang, N. \& Manduca, A. (2010) Calculating tissue shear modulus and pressure by 2D logelastographic methods. Inverse Problems, 26.

Papazoglou, S., Hirsch, S., Braun, J. \& Sack, I. (2012) Multifrequency inversion in magnetic resonance elastography. Phys. Med. Biol., 57, 2329-2346.

Park, E. \& Maniatty, A. M. (2006) Shear modulus reconstruction in dynamic elastography: time harmonic case. Phys. Med. Biol., 51, 3697-.

Sánchez, C., Drapaca, C., Sivaloganathan, S. \& Vrscay, E. (2010) Elastography of biological tissue: direct inversion methods that allow for local shear modulus variations. Image Anal. Recognit., Pt II, 195-206.

Trefethen, L. N. (2000) Spectral Methods in MATLAB. SIAM, Philadelphia, PA.

Tzschätzsch, H., Guo, J., Dittmann, F., Hirsch, S., Barnhill, E., Jöhrens, K., Braun, J. \& Sack, I. (2016) Tomoelastography by multifrequency wave number recovery from time-harmonic propagating shear waves. Medical Image Analysis, 30, 1-10. 